The Astrophysical Journal, 485:447-459, 1997 August 20

(C) 1997. The American Astronomical Society. All rights reserved. Printed in U.S.A.

\title{
EVIDENCE OF SUBSTRUCTURE IN THE CLUSTER OF GALAXIES A3558
}

\author{
Christine C. Dantas, ${ }^{1}$ Reinaldo R. De Carvalho, ${ }^{2,3}$ Hugo V. Capelato, ${ }^{1}$ and Alain Mazure ${ }^{4}$ \\ Received 1996 December 2; accepted 1997 March 25
}

\begin{abstract}
We investigate the dynamical properties of the cluster of galaxies A3558 (Shapley 8). Studying a region of $1 \mathrm{deg}^{2}\left(\sim 3 \mathrm{Mpc}^{2}\right)$ centered on the cluster cD galaxy, we have obtained a statistically complete photometric catalog with positions and magnitudes of 1421 galaxies (down to a limiting magnitude of $B \sim 21$ ). This catalog has been matched to the recent velocity data obtained by Mazure et al. and from the literature, yielding a radial velocity catalog containing 322 galaxies. We analyze the resulting catalog in search of substructure, using different statistical techniques. This analysis shows that the position/velocity space distribution of galaxies shows significant substructure.

A central bimodal core, detected previously in a preliminary study by Dantas, de Carvalho, \& Capelato and by an analysis of the X-ray emission map by Slezak, Durret, \& Gerbal, is confirmed using the adaptive kernel technique and wavelet analysis. We show that this central bimodal substructure is nevertheless composed of a projected feature, kinematically unrelated to the cluster, plus a group of galaxies probably in its initial merging phase into a relaxed core. The $\mathrm{cD}$ galaxy's velocity offset with respect to the average cluster redshift, reported earlier by several authors, is completely eliminated as a result of our dynamical analysis. The untangling of the relaxed core component also allows a better, more reliable determination of the central velocity dispersion, which in turn eliminates the " $\beta$ problem" for A3558. The cluster also shows a "preferential" distribution of subclumps coinciding with the direction of the major-axis position angle of the $\mathrm{cD}$ galaxy and of the central X-ray emission ellipsoidal distribution, in agreement with the anisotropic merger scenario described by West.
\end{abstract}

Subject headings: catalogs - galaxies: clusters: individual (A3558) - galaxies: distances and redshifts galaxies: elliptical and lenticular, $\mathrm{cD}$ - galaxies: photometry

\section{INTRODUCTION}

Clusters of galaxies stand as the ideal probes to test the presently accepted hierarchical scenario of structure formation. According to this picture, if $\Omega \sim 1$, rich clusters are still being formed by mergers of individual groups of galaxies from the field (Richstone, Loeb, \& Turner 1992). Recent optical and X-ray observations seem to be in agreement with that hypothesis, since substructure is found in a large fraction of clusters $(\sim 40 \%-75 \%$; see Bird 1994; West 1994b, and references therein). Recent simulations (e.g., Katz \& White 1993) based on the hierarchical scenario are also beginning to show features with properties that can be associated with substructures found in real clusters.

Apart from the cosmological point of view, the complexity of the multicomponent environment (gas, galaxies, and dark matter) that constitutes a cluster of galaxies can provide important clues about the formation and evolution of individual galaxies. It is especially important to investigate these structural and evolutionary trends within substructures, where galaxy alterations (morphological type, size, and mass distribution) within the denser substructure environment might be more clearly untangled (e.g., Whitmore 1990).

\footnotetext{
${ }^{1}$ Divisão de Astrofisica, Insitutio Nacional de Pesquisas Espacias/ MCT, C.P. 515, 12201-970 São José dos Campos, SP, Brazil.

2 Present address: Department of Astronomy, California Institute of Technology, Pasadena, CA 91125.

${ }^{3}$ On leave of absence from Departamento de Astrofisica, Observatório Nacional, CNPq, Brazil.

${ }^{4}$ Laboratoire d'Astronomie Spatiale, IGRAP, Traverse du Siphon, F-13012 Marseille, France.
}

There are several statistical techniques now available to quantify substructures in clusters. One of the first systematic works was carried out by Geller \& Beers (1982). Using only two-dimensional projected data, they found that $\sim 40 \%$ of the clusters in their sample showed statistically significant substructures. Later, Dressler \& Shectman (1988) developed a statistic based on both position and velocity information and found similar results when applying their test to a sample of 15 clusters. However, in a subsequent study, West \& Bothun (1990) reached opposite conclusions. Bird (1994) has analyzed the several proposed methods and statistics in detail and concluded that $\sim 50 \%$ of the studied sample (33 clusters) contains substructures. More recently, Kriessler \& Beers (1997) constructed new two-dimensional contour maps using Dressler's (1980) sample (the same sample analyzed previously by Geller \& Beers 1982). By application of the adaptive kernel technique (Silverman 1986), they found that $57 \%$ of clusters in their sample presented significant substructure.

The goal of this work is to investigate the structural and dynamical properties of the individual cluster of galaxies A3558 (Shapley 8), in terms of substructure analysis. This cluster has been an object of extensive optical and X-ray observations in the past few years (e.g., Metcalfe, Godwin, \& Spenser 1987; Teague, Carter, \& Gray 1990; Bardelli et al. 1994, 1996; Markevitch, Sarazin, \& Henriksen 1996; Dantas, de Carvalho, \& Capelato 1994; Dantas 1996). This cluster, classified as richness class 4 in the ACO catalog (Abell, Corwin, \& Olowin 1989) and dominated by a central giant $\mathrm{cD}$ galaxy, has attracted much interest, first, due to its location - at the core of the Shapley concentration, a supercluster composed of 25 clusters of galaxies and rich in X- 
ray-emitting clusters (see, e.g., Raychaudhury et al. 1991; Zucca et al. 1993) - and, second, because of its dynamical complexity, due to the presence of substructure (e.g., Bardelli et al. 1996).

Some authors have reported that the central cD galaxy of A3558 has a peculiar velocity relative to the cluster average velocity (Gebhardt \& Beers 1991). This "offset," also found in several cD clusters, was shown by Bird (1994) to be strongly correlated to the presence of substructure in the cluster. Using the KMM method (see, e.g., Ashman, Bird, \& Zepf 1995) in order to assign galaxies to their most probable substructure, Bird concluded that the velocity offset remained only in two out of 25 analyzed clusters (including A3558). Later, Bardelli et al. (1996) partitioned their A3558 velocity sample into two subsamples and verified that the $\mathrm{cD}$ velocity was consistent with the average velocity of one of the subsamples, but the detailed relation of the original offset to the nature of the dynamical status of the central core was never made clear.

Also, one more component of uncertainty in A3558 is its apparently "extreme" $\beta$-discrepancy, as described by Bardelli et al. (1996), which could not be accounted for with the proposed correction of Bahcall \& Lubin (1994). The $\beta$-problem, first noted by Gorenstein et al. (1978) and Jones \& Forman (1984), can be stated in its simple version (see details in, e.g., Gerbal, Durret, \& Lachièze-Rey 1994) as an incompatibility between the observed X-ray slope of the hot intracluster gas and the theoretical temperature ratio of galaxies to gas. This problem has often been referred to in the literature, and some solutions have been proposed (e.g., Bahcall \& Lubin 1994; Gerbal et al. 1994).

In this paper, we resolve the $\mathrm{cD}$ offset and the $\beta$-problem in A3558, using a careful and detailed substructure analysis of a statistically complete velocity sample of galaxies. We present the data reduction and methodology in $\S 2$. In $\S 3$, we briefly review the current available statistical techniques to look for substructure, and apply them to A3558 in $\S 4$. In $\S 5$, we discuss the results and a comparison of all the methods and their significance. We use $q_{0}=0.1$ and $H_{0}=$ $75 \mathrm{~km} \mathrm{~s}^{-1} \mathrm{Mpc}^{-1}$ throughout.

\section{DATA REDUCTION AND METHODOLOGY}

\subsection{Data}

The Abell cluster A3558 (Shapley 8) has been extensively observed in the last 5 years (e.g., Bardelli et al. 1994). It is at a distance of $\sim 14,400 \mathrm{~km} \mathrm{~s}^{-1}$ and is located at the core of the Shapley concentration, a supercluster composed of 25 clusters of galaxies. This cluster is classified as richness class 4 in the ACO catalog (Abell et al. 1989) and is dominated by a central giant $\mathrm{cD}$ galaxy. The cluster seems to be relatively rich in elliptical/lenticular galaxies (Metcalfe, Godwin, \& Peach 1994). All this is suggestive of a regular, well-relaxed, rich cluster of galaxies. We explore this hypothesis by analyzing both the projected distribution of galaxies and its velocity field. Extensive spectroscopic surveys of clusters of galaxies (e.g., the ESO surveys, the Canadian Network for Observational Cosmology survey) have been carried out recently. However, a well-defined magnitude-limited catalog is of fundamental importance for a follow-up spectroscopic study, so that the investigation of the physical properties of clusters becomes meaningful.

We have obtained two digitized images of A3558, one with resolution of $11^{\prime \prime} 67 \mathrm{pixel}^{-1}$ and the other with $1^{\prime \prime}$ pixel $^{-1}$ (Lasker et al. 1990), through scans of the deep IIIa-J plates available at STScI. ${ }^{5}$ Measurement of photometric attributes are improved by the use of high-resolution images, but the noise contribution proportionally increases in the scanning process. For this reason, we tested the confidence limits of the final photometric catalog by analyzing both images of A3558 (defined by a field of $1^{\circ} \times 1^{\circ}$, centered on the $\mathrm{cD}$ galaxy).

\subsection{Detection and Classification with FOCAS}

The data reduction was performed using the FOCAS package (Valdes 1982). Several tests were carried out for both images and for a simulated cluster image, in order to understand how the output catalog responded to variations in the object detection parameters (Dantas 1996). These tests showed that the image with $1^{\prime \prime}$ pixel $^{-1}$ resolution provided higher quality information, being a compromise between noisier measurement and better sampling. We defined a minimum detection area of 65 pixels $^{2}$, corresponding to the area of a circle with radius equal to $\sim 3$ times the typical seeing disk at Siding Spring. Detection was performed at a threshold level of 3 times the sky rms. This corresponds on average to $7 \%$ of the local sky value. Besides the detection procedure, an important step in building a reliable catalog is to set the point-spread function (PSF) that is to be used in the object classification procedure. A specific program in FOCAS automatically searches for stellar and nonsaturated objects for the construction of the PSF. In this work, we limited that search for stars to the magnitude interval $B=16-19 \mathrm{mag}$. The stars selected by FOCAS were visually inspected, and after pruning asymmetric objects, we then recomputed the PSF. The classification of objects was then performed according to specific rules in FOCAS that we kept at their default values (see Valdes 1982 for a detailed description). After this process, a visual inspection was carried out to eliminate cataloged objects that were clearly misclassified by FOCAS. In general, these objects were overlapping stars and/or galaxies forming a unique diffuse object mistaken for a galaxy. This type of contamination is estimated to be $\sim 10 \%$ of the whole catalog (see also de Carvalho, Ribeiro, \& Zepf 1994).

\subsection{Magnitude Calibration and Limiting Magnitude}

The calibration of the magnitude scale was accomplished using CCD photometry from Postman \& Lauer (1995, hereafter PL) and Melnick \& Quintana (1984, hereafter MQ). PL obtained their data through actual photometric measurements of the cD galaxy (Johnson $B$ system) whereas the data from MQ are a compilation of measurements from the literature (in different passbands). Based on this, we decided to use the data from PL for actual calibration and to use the MQ data as a consistency test. Calibration was done by estimating the difference between the magnitude listed by PL and MQ and the instrumental total magnitude. FOCAS calculates the total luminosity by integrating the flux inside the "total area" of the object. This "total area" is in turn determined by fitting the concavities in $(x, y)$ of the isophote shape and then adding rings around the object up to 2 times

\footnotetext{
${ }^{5}$ Photometry obtained using the Guide Stars Selection System Astrometric Support Program, developed at the Space Telescope Science Institute (STScI is operated by the Association of Universities for Research in Astronomy, Inc., for NASA).
} 
the detection area (called the "grown area"). Consequently, the FOCAS instrumental magnitude beyond the grown radius is constant. In this case PL provide measurements beyond the radius corresponding to the total area. For this reason, we used the task APPHOT in IRAF to measure the instrumental magnitude within specific apertures. The internal apertures were not used, because of saturation of the $\mathrm{cD}$ galaxy. The zero point of the magnitude scale resulted in $K=32.86 \pm 0.16 \quad$ (using data from PL) and $K=32.92 \pm 0.18$ (using data from MQ). The comparison between the photometry results of PL, MQ, and this work is shown in Figure 1 (top), with corresponding residuals (bottom).

The limiting magnitude of the catalog $\left(B_{\text {lim }} \sim 21\right)$ was first estimated using the magnitude histogram of all detected objects not classified by FOCAS as "noise" objects and was defined by the last bin prior to a significant counting decrease (Picard 1991). This magnitude-limit estimate will be reanalyzed in the next section.

\subsection{Final Galaxy Catalog}

The photometric catalog described above was obtained by running FOCAS under its default classification rules, by which the detected objects are assigned to five predefined "resolution" classes: (n) noise, (s) star, (sf) fuzzy star, (g) galaxy, and (d) diffuse. A further class, "long," is defined for those objects presenting flat and very elongated geometries. As discussed in detail by Valdes (1982), the uncertainties of such a classification are due, for one part, to the natural uncertainties in the partitioning of the image parameter space into astronomical classes and, for the other, to the rapid decrease of the classification probabilities at fainter magnitudes.

In order to circumvent these problems, which are indeed common to most automated astronomical image classifiers, we used a sample of 325 spectroscopically confirmed galaxies to provide an independent check on the FOCAS classifier's performance. This sample was obtained by

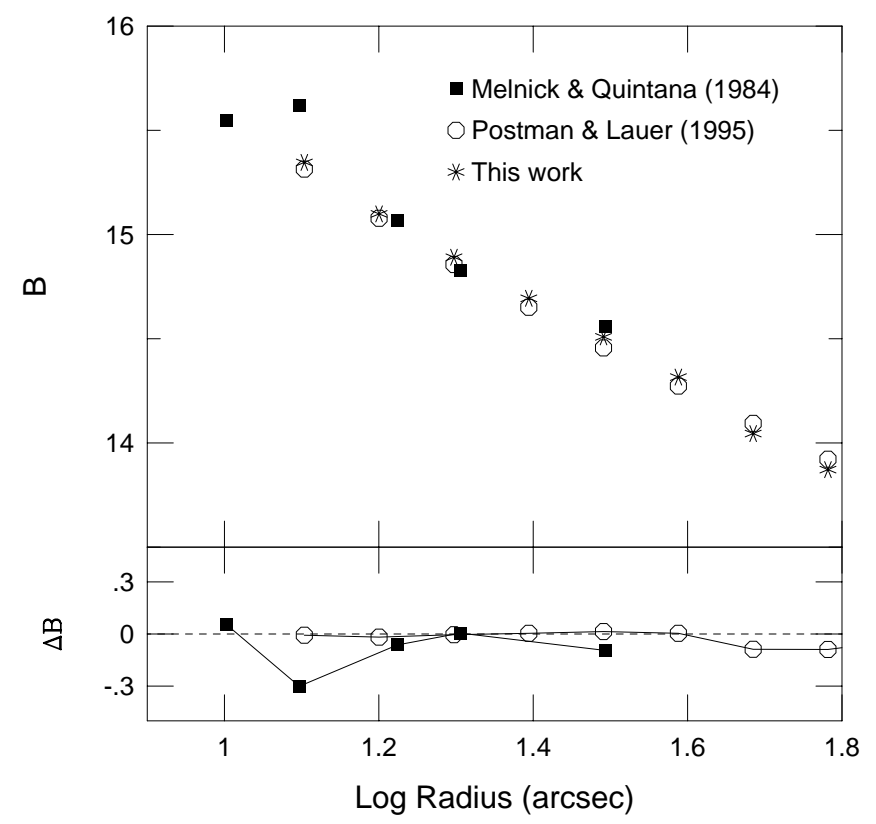

Fig. 1.-Comparison between the photometry results of PL, MQ, and this work, with corresponding residuals (where $\Delta B$ is the difference between our results and those of PL and MQ).
TABLE 1

Probability of Galaxy Classification in Given Resolution Class

\begin{tabular}{cccccc}
\hline \hline$\Delta B$ & $\begin{array}{c}P(\mathrm{~s} \mid \mathrm{g}) \\
(\%)\end{array}$ & $\begin{array}{c}P(\mathrm{sf} \mid \mathrm{g}) \\
(\%)\end{array}$ & $\begin{array}{c}P(\mathrm{~g} \mid \mathrm{g}) \\
(\%)\end{array}$ & $\begin{array}{c}P(\mathrm{~d} \mid \mathrm{g}) \\
(\%)\end{array}$ & $\begin{array}{c}P(\text { long } \mid \mathrm{g}) \\
(\%)\end{array}$ \\
\hline $14-18 \ldots \ldots$ & 1 & 0 & 98 & 0.5 & 0 \\
$18-21 \ldots \ldots$ & 1 & 12 & 86 & 0 & 1 \\
\hline
\end{tabular}

compiling the redshift catalogs of Bardelli et al. (1994), Mazure et al. (1996), Metcalfe et al. (1987), MQ, Stein (1996), and Teague et al. (1990). By cross-correlating it with our photometric catalog, we produced a "matched" catalog consisting of 322 galaxies. For the remaining three galaxies, two were not included in the matched catalog because they were identified as multiple systems not split by FOCAS, and the other one could not be identified with any detected object. The statistics of FOCAS classification of these 322 objects is given in Table 1 (note that no galaxies were classified as " $n$ "). As can be seen, the bright galaxies were correctly classified as "g," with a negligible contamination of the other classes. In contrast, faint galaxies are increasingly classified as "sf." In view of these results, we conservatively decided to keep in the final catalog only objects classified as "g" and "sf." This final galaxy catalog, with 1421 galaxies, is published in its entirety in computerreadable form in the AAS CD-ROM Series, Volume 9. We found, using the same methodology as Picard (1991), that the limiting magnitude of our catalog of galaxies is about the same as that of the initial photometric catalog $(\S 2.3)$, that is, $B_{\text {lim }} \sim 21$.

We have carried out a similar completeness analysis for the subset of galaxies with measured velocities. We found that it is only moderately complete in all magnitude ranges (see Fig. 2). Nevertheless, the completeness level per magnitude interval increases for galaxies systematically closer in projection to the central cD galaxy. For instance, an $\sim 88 \%$

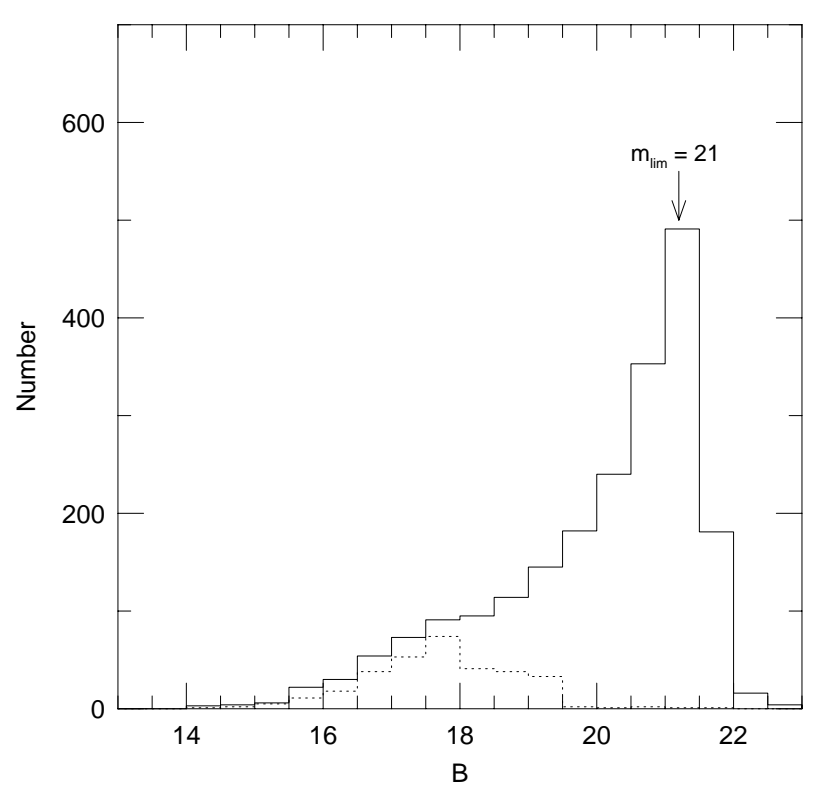

FIG. 2.-Magnitude histograms of the photometric catalog (solid line), including all detected objects classified as "g" and "sf," and the velocitymatched catalog with 322 galaxies (dotted line). The magnitude limit based on Picard's (1991) methodology is marked with an arrow. 
cumulative completeness level up to the $17.5-18 \mathrm{mag}$ bin is achieved for the galaxies within a $900^{\prime \prime}$ radius around the $\mathrm{cD}$, whereas a $\sim 95 \%$ level up to the $18-18.5 \mathrm{mag}$ bin is found using a $500^{\prime \prime}$ radius. These features are due to the inhomogeneities of the assembled velocity sample and will be taken into account in the dynamical analysis.

\section{VELOCITY DISTRIBUTION}

We have used the robust estimators discussed by Beers, Flynn, \& Gebhardt (1990) to establish the membership of the final catalog of galaxies with measured velocities $(322$ galaxies) and to obtain the global kinematic parameters of A3558. A histogram of all measured velocities for the matched catalog (Fig. 3, inset) was first used to eliminate the obvious foreground and background objects $(v<9000 \mathrm{~km}$ $\mathrm{s}^{-1}$ and $v>18,000 \mathrm{~km} \mathrm{~s}^{-1}$ ). We then used the ROSTAT code (Beers et al. 1990, 1991) to compute the $C_{\mathrm{BI}}$ and $S_{\mathrm{BI}}$ estimators for the remaining velocities and recursively eliminated the galaxies that were 3 biweighted scales from $C_{\text {BI }}$ (Teague et al. 1990), which converged to a membership criterion of $12,000 \mathrm{~km} \mathrm{~s}^{-1}<v<18,000 \mathrm{~km} \mathrm{~s}^{-1}$. The final "velocity" catalog contains 282 possible member galaxies brighter than $B_{\text {lim }} \sim 21$. Its velocity histogram is shown in Figure 3. For comparison, the velocity histogram of galaxies within a $900^{\prime \prime}$ radius circle around the $\mathrm{cD}$ galaxy is shown in Figure 4. As argued in the previous section, this central subset $\left(R<900^{\prime \prime}\right)$ is $\sim 88 \%$ complete up to the 18 mag bin and may be taken as a representative sample of the core of A3558.

At this point we should note that the $1^{\circ} \times 1^{\circ}$ field covered by our photometry also includes, other than A3558 itself, the poor cluster SC 1327-312 (R.A.[2000.0] $=13^{\mathrm{h}} 30^{\mathrm{m}} 04^{\mathrm{s}}, \quad$ decl. $[2000.0]=-31^{\circ} 44^{\prime} 49^{\prime \prime}$ ), first noted as an X-ray source by Breen et al. (1994). The
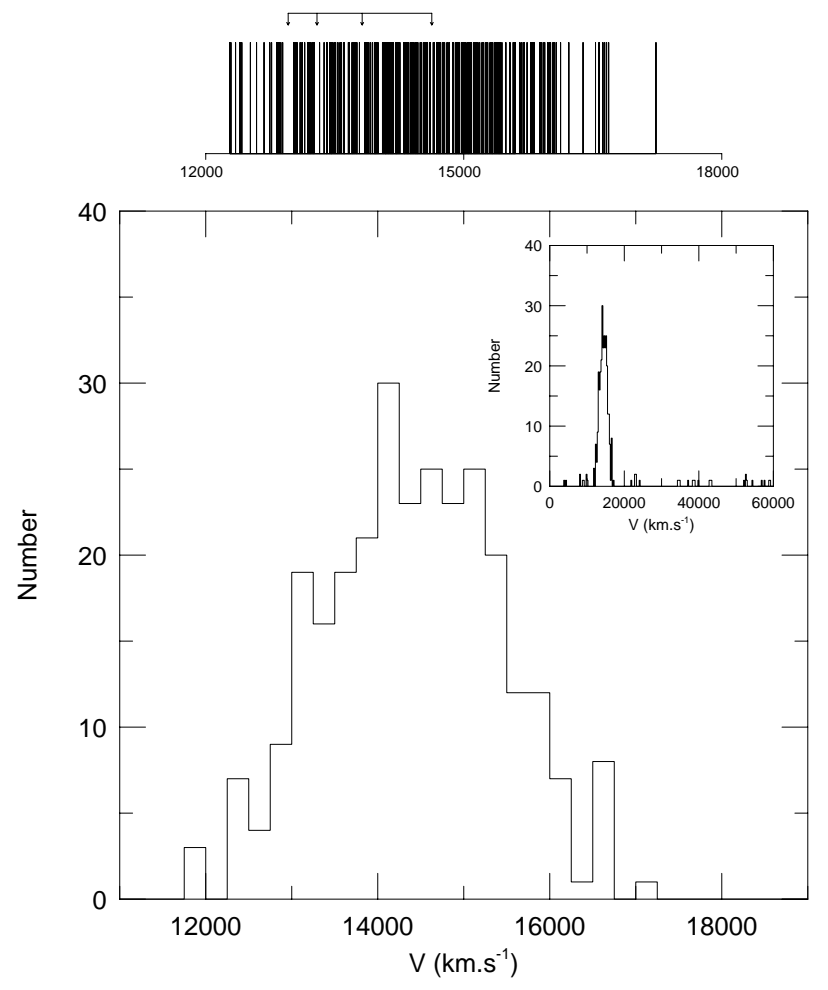

FIG. 3.-Velocity histogram for the final $3 S_{\mathrm{BI}}$-clipped matched catalog with 282 objects. Inset: Velocity histogram of the matched catalog with 322 objects. Top: "Stripe" density plot for the velocity distribution; gaps are indicated by arrows.

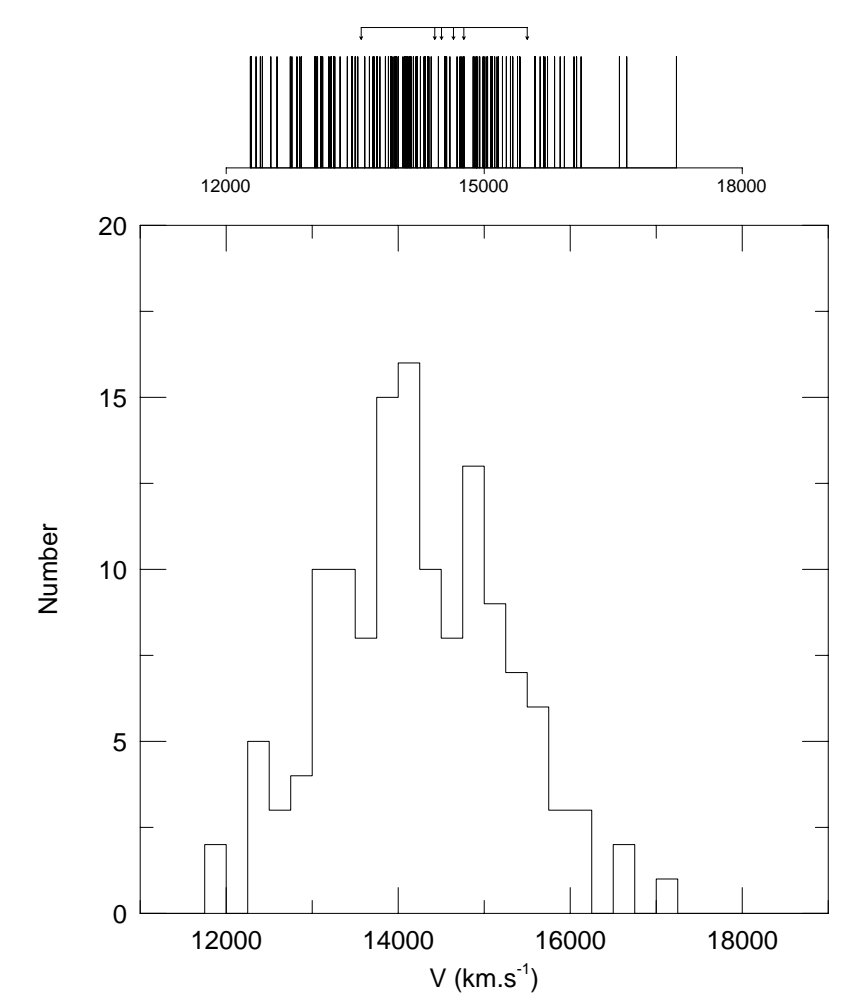

FIG. 4.-Velocity histogram for the $R<900$ " sample. Top: "Stripe" density plot for the velocity distribution; gaps are indicated by arrows.

velocity histogram of galaxies inside a circular region of $400^{\prime \prime}$ radius centered at the peak X-ray emission of SC 1327-312 (Bardelli et al. 1996) is displayed in Figure 5. This sample, also defined for galaxies in the velocity range $12,000 \mathrm{~km} \mathrm{~s}^{-1}<v<18,000 \mathrm{~km} \mathrm{~s}^{-1}$, contains 17 galaxies with measured velocities and is $82 \%$ complete down to $18 \mathrm{mag}$.

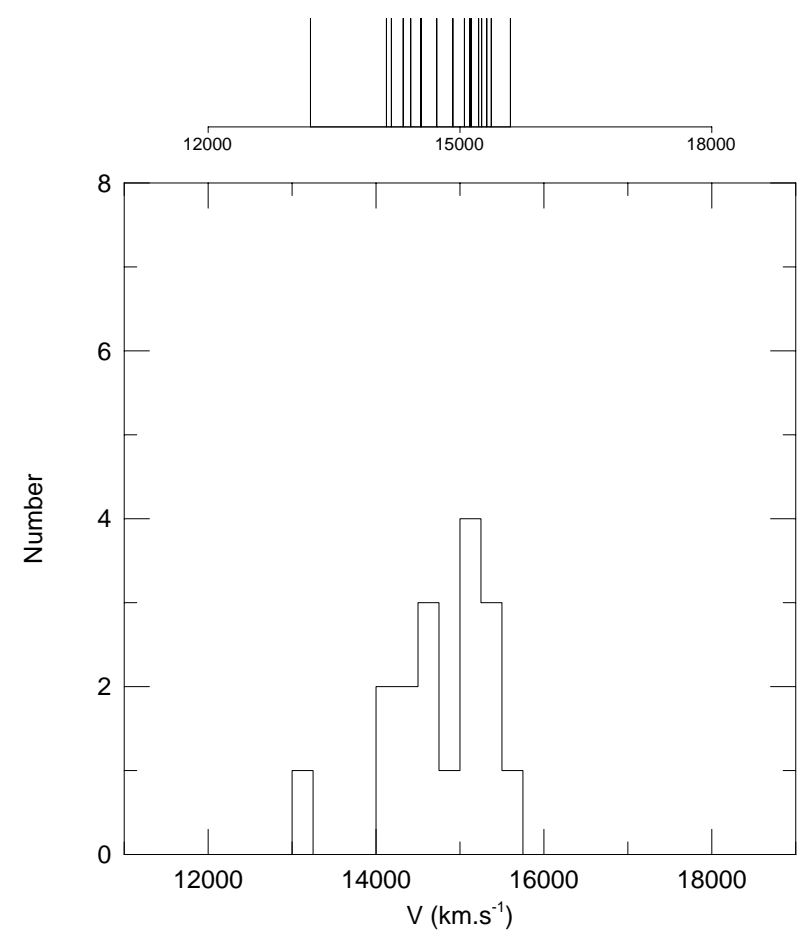

FIG. 5.-Same as Fig. 4, but for the SC 1327-312 sample 
In Table 2, the global kinematic parameters of the three samples discussed above are presented. The columns are as follows: (1) sample; (2), (3) $C_{\mathrm{BI}}$ and $S_{\mathrm{BI}}$ (in $\mathrm{km} \mathrm{s}^{-1}$ ), respectively, with their $90 \%$ confidence intervals; (4) numbers of galaxies used for the estimate; and (5) source reference. We will assume the redshift corresponding to the central $\left(R<900^{\prime \prime}\right)$ region as that representative for the cluster: $z=0.0475 \pm 0.0003$.

We have performed several normality tests for the velocity distribution samples defined above using the ROSTAT code. The results indicate that the velocity distribution of A3558, using all 282 probable member galaxies, is consistent with normality under most of the statistical tests included in the ROSTAT routine (significance levels for the null hypothesis greater than $25 \%$ ). The scaled tail index, $\mathrm{TI}=0.983$, indicates that the sample distribution is close to Gaussian, whereas the asymmetry index, $\mathrm{AI}=0.971$, suggests that the distribution is skewed toward higher velocities (for a thorough discussion on these two indexes, see Bird \& Beers 1993). Similarly, we find that the "core" $R<900^{\prime \prime}$ sample (Fig. 4) is also approximately Gaussian, with $\mathrm{TI}=0.942$ and $\mathrm{AI}=0.996$. The Dip test indicates that the probability of nonunimodality of the "core" sample is very large $(\sim 83 \%)$, and this result is in accordance with the presence of a bimodal core, as reported in detail in the next section. The SC 1327-312 sample (see Fig. 5) displays an asymmetric distribution, $\mathrm{AI}=-0.669$. We find $\mathrm{TI}=0.768$ for this sample, which indicates that the tails are probably underpopulated, if the parent distribution is indeed Gaussian, and the Dip test indicates that the probability of nonunimodality is reasonably high $(\sim 70 \%)$.

In order to discuss the importance of gaps in velocity space, we have followed the weighted gap analysis presented by Beers et al. (1992). A single gap is considered significant if its Gaussian-weighted value is greater than 2.25. Significant gaps found by sampling a Gaussian distribution occur with a frequency of less than $3 \%$, independently of the sample size. The "total gap probability" is another indicator of gap significance: it is the cumulative probability of finding a number $N$ of individually significant gaps anywhere in the distribution. For instance, a low total gap probability indicates that, after resampling, all the $N$ original gaps will very unlikely be found anywhere in the distribution, that is, the $N$ gaps will be collectively significant. We use the "stripe" density diagram to show the location of each significant gap (Beers et al. 1992). Each galaxy in velocity space is indicated

TABLE 2

Global Kinematic Parameters of Major Structures in the Field AROUND A3558

\begin{tabular}{clcrc}
\hline \hline $\begin{array}{c}\text { Sample } \\
(1)\end{array}$ & \multicolumn{1}{c}{$\begin{array}{c}C_{\mathrm{BI}} \\
(2)\end{array}$} & \multicolumn{1}{c}{$\begin{array}{c}S_{\mathrm{BI}} \\
\left(\mathrm{km} \mathrm{s}^{-1}\right) \\
(3)\end{array}$} & $\begin{array}{c}N_{\text {gal }} \\
(4)\end{array}$ & $\begin{array}{c}\text { Reference } \\
(5)\end{array}$ \\
\hline $\mathrm{A} 3558 \ldots \ldots \ldots \ldots \ldots$ & $14434_{-57}^{+58}$ & $1030_{-43}^{+44}$ & 282 & 1 \\
& $14242 \pm 80$ & $986 \pm 60$ & 150 & 2 \\
& $14233 \pm 91$ & $973_{-66}^{+129}$ & 114 & 3 \\
& $14306_{-165}^{+185}$ & $923_{-102}^{+120}$ & 80 & 4 \\
& $14237 \pm 150$ & $991^{+157}$ & 50 & 5 \\
& $14628 \pm 150$ & $1044_{-162}^{+298}$ & 13 & 6 \\
& $14238_{-94}^{+93}$ & $1016_{-64}^{+63}$ & 133 & 1 \\
$\mathrm{SC} 1327-312 \ldots \ldots$. & $14841_{-140}^{+139}$ & $580_{-118}^{+119}$ & 16 & 1
\end{tabular}

NoTE.-Quoted uncertainties represent $90 \%$ confidence intervals.

REFERENCES.- (1) This work; (2) Bardelli et al. 1996; (3) Teague et al. 1990; (4) Bird 1994; (5) Metcalfe et al. 1987; (6) Melnick \& Quintana 1981. by a unique vertical line, and significant gaps are indicated by arrows.

In the 282 galaxy sample velocity distribution (see Fig. 3, top), we find that the probability of each individual gap is 0.03 , except for one gap at $\sim 13,700 \mathrm{~km} \mathrm{~s}^{-1}$, which is 0.002 . Nevertheless, the "total gap probability" indicates that the probability that all these gaps occur anywhere in the distribution is greater than $\sim 40 \%$. The "core" sample has several gaps. The position, size, weighted size, and probability of each gap for this sample are $13,570 \mathrm{~km} \mathrm{~s}^{-1}(\Delta v=79$ $\left.\mathrm{km} \mathrm{s}^{-1}, z_{*}=2.297, p=0.03\right), 15,501 \mathrm{~km} \mathrm{~s}^{-1}(\Delta v=172 \mathrm{~km}$ $\left.\mathrm{s}^{-1}, z_{*}=2.457, p=0.03\right), 14,505 \mathrm{~km} \mathrm{~s}^{-1}\left(\Delta v=73 \mathrm{~km} \mathrm{~s}^{-1}\right.$, $\left.z_{*}=2.470, p=0.03\right), 14,640 \mathrm{~km} \mathrm{~s}^{-1}\left(\Delta v=83 \mathrm{~km} \mathrm{~s}^{-1}, z_{*}=\right.$ $2.580, p=0.014), 14,425 \mathrm{~km} \mathrm{~s}^{-1}\left(\Delta v=84 \mathrm{~km} \mathrm{~s}^{-1}, z_{*}=\right.$ $2.658, p=0.014)$, and, finally, $14,763 \mathrm{~km} \mathrm{~s}^{-1}(\Delta v=105 \mathrm{~km}$ $\mathrm{s}^{-1}, z_{*}=2.803, p=0.006$ ). The "total gap probability" is $\sim 21 \%$ in this case: the occurrence of all these gaps anywhere in the distribution after resampling is relatively small. The SC 1327-312 sample shows no significant gaps.

The presence of gaps indicates that the distribution probably has several "clumps" in the velocity space, but their overlapping makes separation impossible without positional information. A complete substructure analysis, using velocity/position data, is presented in the following section.

\section{SUBSTRUCTURE ANALYSIS}

\subsection{Introduction}

Clusters of galaxies can be morphologically arranged into a one-dimensional sequence (from irregular to regular) that suggests a direction of dynamical evolution (from young to dynamically evolved systems). A virialized cluster is also expected to have a Gaussian line-of-sight velocity profile, whereas a young system should present significant deviations from a Gaussian profile in its velocity distribution. Although separate positional and velocity investigations can yield general clues about the global dynamical status of a cluster, only three-dimensional $(x, y, v)$ diagnostics can reduce the possibility of misinterpretation, since only combined correlations between position and velocity may define physical substructures, suggesting a nonequilibrium state of the cluster. Recent multifiber spectroscopy has allowed the construction of large-scale redshift surveys (Zabludoff et al. 1993; Mazure et al. 1996), which, added to positional and photometric data, contribute to a reliable approach to the problem of substructure in clusters of galaxies (Bird 1994). Indeed, recent optical and X-ray observations (West 1994b and references therein) suggest that up to $75 \%$ of clusters show substructure in their morphologies.

The presence of substructures can also be analyzed via global statistical tests that yield a substructure significance index for the data. These tests are especially useful for a general and direct comparative analysis of a large sample of clusters (see, e.g., Bird 1994), but they do not pinpoint the location, extension, and dynamical nature of the substructures present in the data. For this reason, we shall adopt mapping techniques that are quite useful for this purpose.

\subsection{Substructure Mapping and Dynamical Analysis}

We use the adaptive kernel technique (AK; see Silverman 1986) with a generalized Epanechnikov kernel in order to map the projected density of galaxies and also the local average velocities and the local velocity dispersion of galaxies (Biviano et al. 1996). Following Biviano et al., we have 
obtained significance maps for these quantities by taking the average of 1000 bootstraps of the original cluster and subtracting the corresponding $3 \sigma$ maps from this average. Structures that can "survive" this subtraction are statistically significant features at the $3 \sigma$ significance level.

We have divided our field into three regions, namely, the core region, defined by a square field of $1000^{\prime \prime}$ side centered on the cD galaxy and encompassing most of the X-ray emission of the cluster-in this region the velocity sample is $89 \%$ complete down to $B \sim 19$; an intermediate region defined by a square field of $1800^{\prime \prime}$ side centered on the cD galaxy, for which the velocity sample is $88 \%$ complete down to $B \sim 18$; and the whole $1^{\circ} \times 1^{\circ}$ field, for which the velocity sample is not complete at any limiting magnitude. All mappings have grids with $1^{\prime}\left(\simeq 0.05 h_{75}^{-1} \mathrm{Mpc}\right)$ spacing. These AK maps are presented in Figures 6-13, where in all figures the original cluster map is in the top panel and the $3 \sigma$ confidence map in the bottom panel.

In order to analyze the substructures present in these figures, we use the wavelet transform technique (WT; see Grossmann \& Morlet 1987; Slezak, Bijaoui, \& Mars 1990) to generate subcatalogs of galaxies for each substructure of interest (namely, the central substructures and the poor cluster SC 1327-312). These subcatalogs were constructed by selecting galaxies inside a $2 s$ radius around density peaks, where $s$ is the wavelet scale that best characterizes the analyzed structure (for details, see Escalera et al. 1994; Slezak et al. 1990).

The core region (Figs. 10-12) features a significant bimodal structure (denoted by " $\mathrm{A}$ " and " $\mathrm{B}$ " in all maps). This central region is thoroughly analyzed in the following subsections. The apparent central bimodal core is also found in the intermediate-region map (Fig. 6), where several significant substructures (at $3 \sigma$ significance level) are also present. Statistically significant average velocity and velocity dispersion structures can also be noted (Figs. 7 and 8, respectively). The whole field around A3558 is clearly marked with several substructures (Fig. 9). The poor cluster SC 1327-312 toward the southeast, known a priori to be partially represented on our field, is also clearly detected. Unfortunately, the incompleteness of the velocity catalog for the whole region does not allow us to assess the reality of most these substructures. However, as we argue below, their abundance and projected distribution suggest that the infall region of A3558 ( $>1 \mathrm{Mpc}$ ) may be composed of several groups of galaxies in the process of merging.

Indeed, we qualitatively note a "preferential alignment" at the $\sim 45^{\circ}$ position angle of a diverse set of features in this cluster, ranging from small to large scales. An application of the Lee statistics (Fitchett 1988) to this cluster also indicates that the $\sim 45^{\circ}$ direction has a greater probability of bimodality. This alignment coincides with (1) the major-axis position angle of the dominant galaxy, (2) the major-axis position angle of the isocontours of the central core (using AK/WT maps, as well as an X-ray contour map), (3) a marginal velocity gradient direction across the central bimodal substructures, and (4) the direction of alignment of major subclumps, namely, the bimodal core and SC $1327-312$. This alignment seems to persist even beyond the analyzed field. A qualitative inspection of an isopleth map of a roughly $32^{\circ} \times 32^{\circ}$ region around A3558 (see Fig. 2 of Raychaudhury et al. 1991) also indicates an alignment of all major clusters in the Hydra-Centaurus region spanning from position angles of $\sim 40^{\circ}$ to $\sim 60^{\circ}$. Considering the
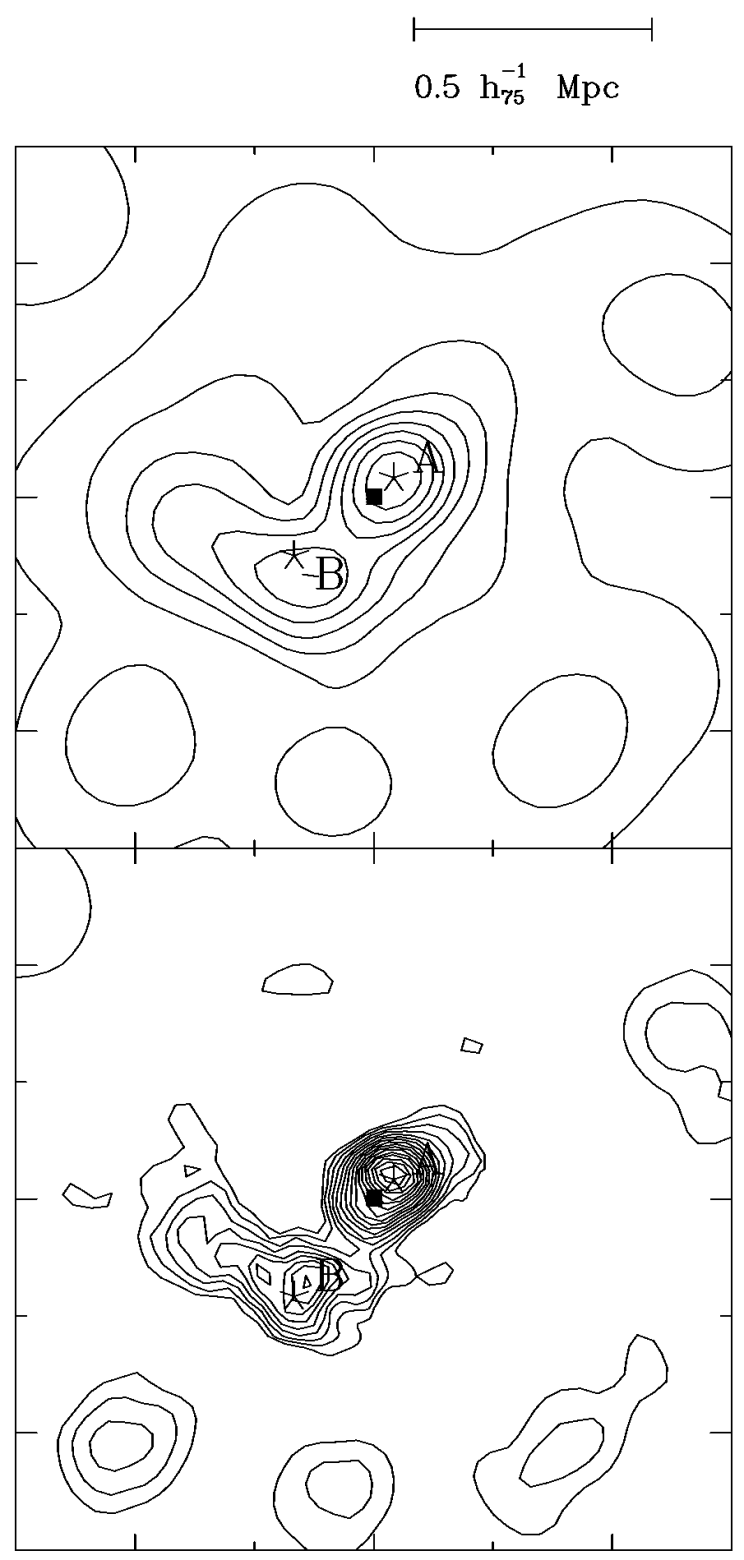

FIG. 6.-Isodensity contour map produced using the adaptive kernel technique (top) and its $-3 \sigma$ significance level map (bottom) for the intermediate region, centered at the $\mathrm{cD}$ galaxy (square). North is up and east is to the left; both axes have ticks equally spaced by $300^{\prime \prime}$. Top, contours run from zero to $2 \times 10^{-4}$ galaxies $\mathrm{deg}^{-2}$ in intervals of $2 \times 10^{-5}$; bottom, contours run from zero to $9.6 \times 10^{-5}$ galaxies $\mathrm{deg}^{-2}$ in intervals of $6 \times 10^{-6}$.

physical dimensions involved, it is quite surprising that the average alignment of major clusters in the Shapley concentration is consistent with the direction of alignment of several substructures within A3558 and with the major-axis position angle of its dominant galaxy. We find that these small- to large-scale "coincidental" associations (namely, $\mathrm{cD}$ major-axis match to the general clustering alignment) can be taken as observational evidence of an anisotropic merger scenario as, for instance, that proposed by West (1994a).

\subsubsection{Complexity of the Central Core of $A 3558$}

Figure 10 (top) presents the projected density contour map of the very central core of A3558. It shows a clear bimodal structure that is very prominent on the $3 \sigma$ con- 


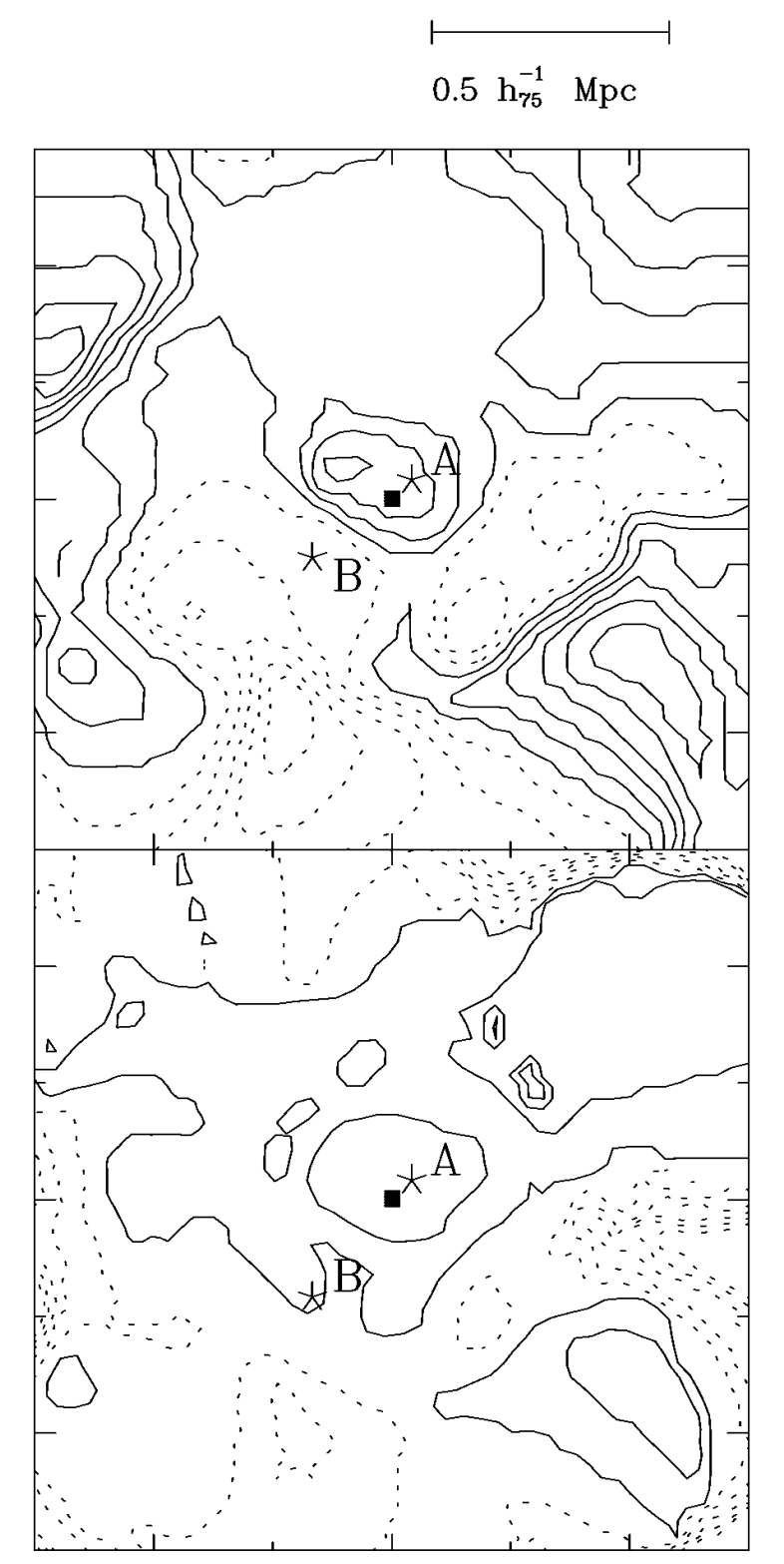

Fig. 7.- Same as Fig. 6, but showing the local average velocity contour map. Top, contours run from 13,100 to $15,600 \mathrm{~km} \mathrm{~s}^{-1}$ in intervals of 200 $\mathrm{km} \mathrm{s}^{-1}$; bottom, contours run from 10,000 to $14,200 \mathrm{~km} \mathrm{~s}^{-1}$ in intervals of $600 \mathrm{~km} \mathrm{~s}^{-1}$

fidence map, as can be seen in the bottom panel of this figure. The two central clumps are labeled "A" (R.A. $=13^{\mathrm{h}} 27^{\mathrm{m}} 49^{\mathrm{s}}, \quad$ decl. $=-31^{\circ} 28^{\prime} 59^{\prime \prime}$ ) and “B” (R.A. $=13^{\mathrm{h}} 28^{\mathrm{m}} 13^{\mathrm{s}}$, decl. $=-31^{\circ} 33^{\prime} 10^{\prime \prime}$ ) in Table 3. The average velocities of these central substructures are also

TABLE 3

Characteristics and Kinematic Parameters of the Central Core Region Substructures

\begin{tabular}{cccr}
\hline \hline Structure & $N_{\text {gal }}$ & $\begin{array}{c}C_{\mathrm{BI}} \\
\left(\mathrm{km} \mathrm{s}^{-1}\right)\end{array}$ & $\begin{array}{c}S_{\mathrm{BI}} \\
\left(\mathrm{km} \mathrm{s}^{-1}\right)\end{array}$ \\
\hline $\mathrm{A} \ldots \ldots \ldots \ldots \ldots \ldots \ldots$ & 24 & $14732 \pm 231$ & $1142_{-140}^{+141}$ \\
$\mathrm{~A}^{\prime} \ldots \ldots \ldots \ldots \ldots \ldots \ldots$ & 13 & $15565 \pm 176$ & $587_{-145}^{+144}$ \\
$\mathrm{~B} \ldots \ldots \ldots \ldots \ldots \ldots \ldots$ & 25 & $14012 \pm 157$ & $897_{-116}^{+115}$ \\
$\mathrm{Core}-\mathrm{A}^{\prime} \ldots \ldots \ldots \ldots$ & 65 & $14022 \pm 103$ & $769 \pm 72$ \\
Core $-\mathrm{A}^{\prime}-\mathrm{B} \ldots \ldots \ldots$ & 40 & $14035 \pm 113$ & $683 \pm 96$ \\
\hline
\end{tabular}

NoTE.-Quoted uncertainties represent $90 \%$ confidence intervals.

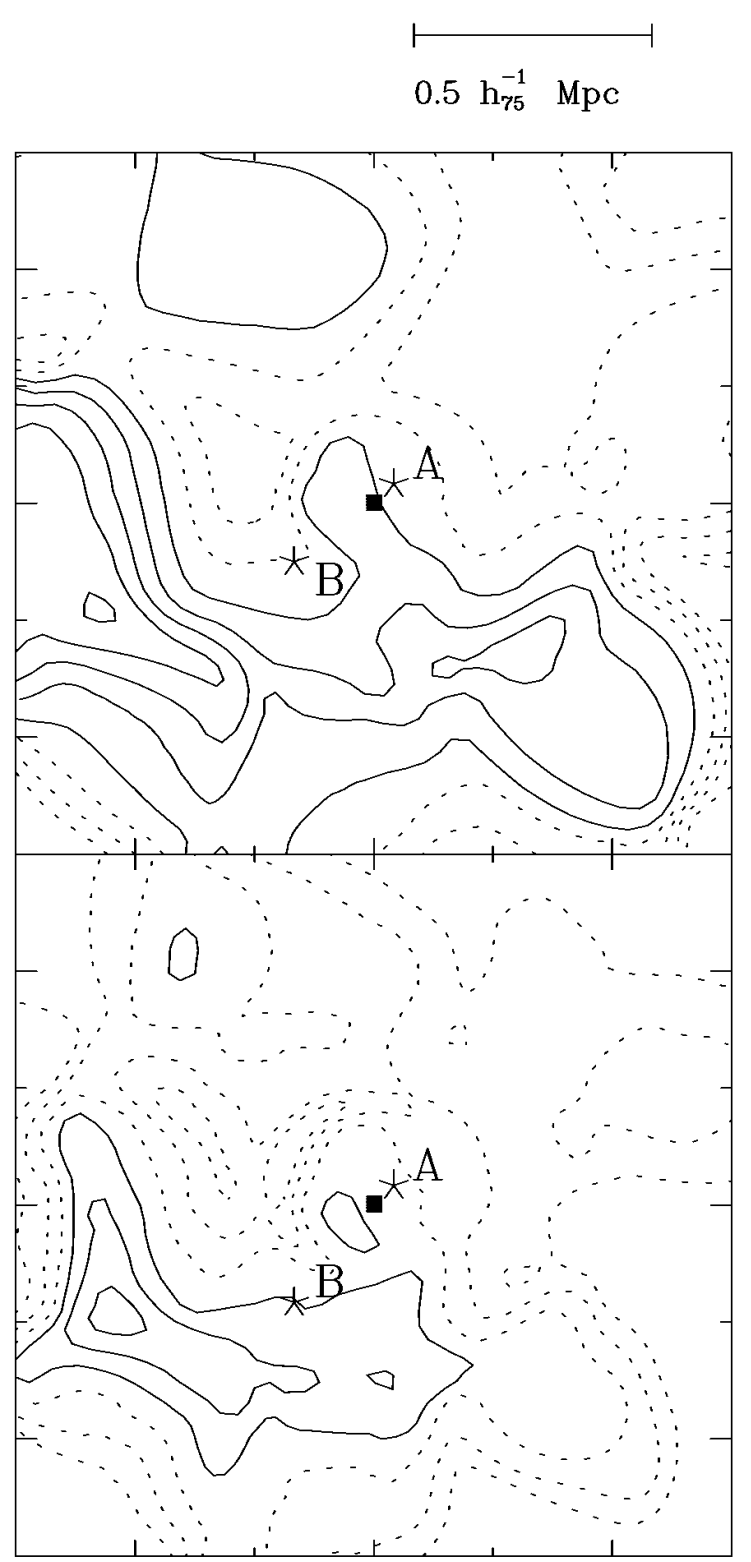

FIG. 8.- Same as Fig. 6, but showing the local velocity dispersion contour map. Top, contours run from 0 to $1800 \mathrm{~km} \mathrm{~s}^{-1}$ in intervals of 200 $\mathrm{km} \mathrm{s}^{-1}$; bottom, contours run from 0 to $1000 \mathrm{~km} \mathrm{~s}^{-1}$ in intervals of $200 \mathrm{~km}$ $\mathrm{s}^{-1}$.

significantly distinct, as can be seen in Figure 11 and also by an inspection of the $C_{\mathrm{BI}}$ values displayed in Table 3 (the difference of their average velocities are out their $90 \%$ interval on central location). The isodispersion map (Fig. 12) indicates that the average velocity dispersion of substructure A differs from that of the central region, although not very significantly. This is also in accordance with the $S_{\mathrm{BI}}$ values listed in Table 3 .

Figure 13 displays the projected density contour map of the faint galaxy subsample of the core region $(19<B<21)$. The distribution of faint galaxies is much smoother than that of the brighter galaxies, having peak densities that attain no more than half of those of the bright subsample. Both substructures A and B are still present in this map, but much less prominently. There are also hints of new significant clumps that are mapped only by faint galaxies. Unfortunately, this faint central subsample contains only five 


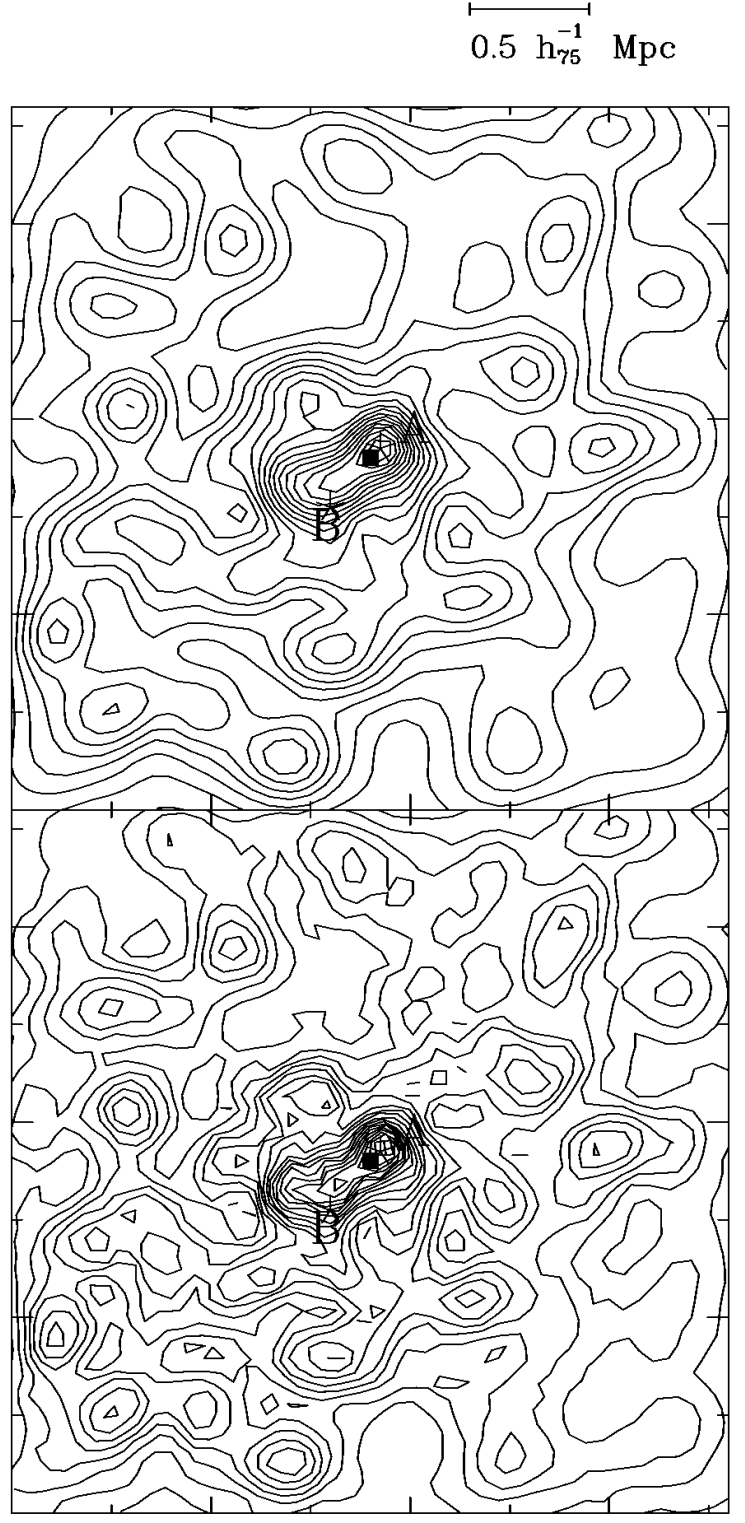

FIG. 9.-Isodensity contour map (top) and its $-3 \sigma$ significance level map (bottom) for the whole field of A3558 $\left(B_{\text {lim }}=19\right)$. North is up and east is to the left; both axes have ticks equally spaced by $500^{\prime \prime}$. Top, contours run from zero to $4 \times 10^{-4}$ galaxies $\mathrm{deg}^{-2}$ in intervals of $2 \times 10^{-5}$; bottom, contours from zero to $2.6 \times 10^{-4}$ galaxies $\mathrm{deg}^{-2}$ in intervals of $1.3 \times 10^{-5}$.

galaxies with measured velocities (all in the range 12,000 km $\mathrm{s}^{-1}<v<18,000 \mathrm{~km} \mathrm{~s}^{-1}$ ), precluding a more detailed analysis about the possible existence of background clusters.

The above discussion stresses the apparent complexity of the central core of A3558. This is to be contrasted with the featureless X-ray emission (Fig. 14), which is centered on the $\mathrm{cD}$ galaxy with a smooth extension toward the location of substructure B (see also the detailed study by Bardelli et al. 1996). This suggests that the gas is driven by a smooth gravitational potential that does not seem to be mapped by the bright galaxy component of the cluster. We are thus led to conjecture that the projected core of A3558 is composed of three dynamically distinct structures: the main virialized core of the cluster, which is traced by the X-ray gas and maybe also by the faint galaxy component, and the galaxy clumps A and B described above.

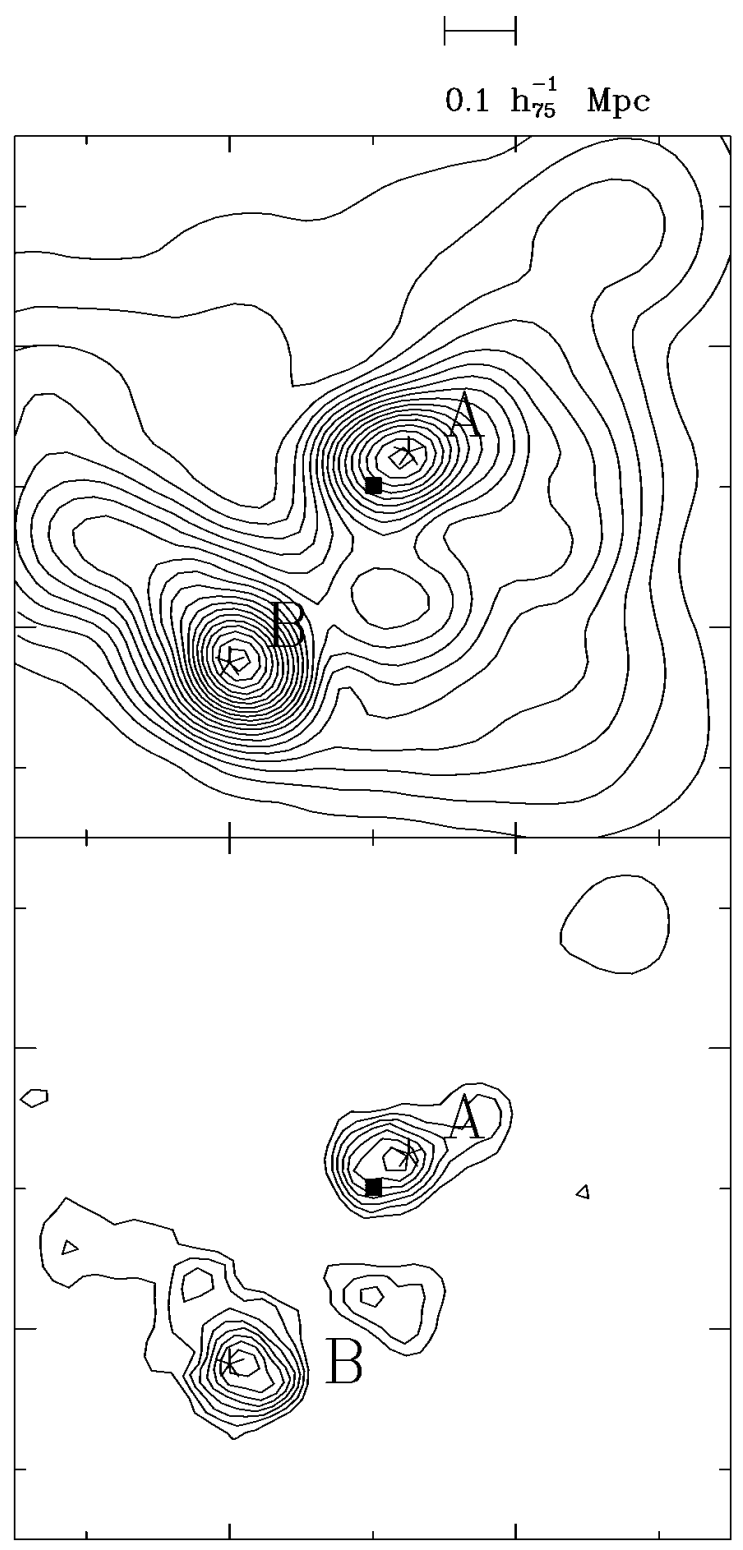

FIG. 10.-Isodensity contour map (top) and its $-3 \sigma$ significance level map (bottom) for the central core region, centered at the $\mathrm{cD}$ galaxy (square). North is up and east is to the left; both axes have ticks equally spaced by $200^{\prime \prime}$. Top, contours run from zero to $4 \times 10^{-4}$ galaxies $\mathrm{deg}^{-2}$ in intervals of $2 \times 10^{-5}$; bottom, contours run from zero to $1.4 \times 10^{-4}$ galaxies $\mathrm{deg}^{-2}$ in intervals of $2 \times 10^{-5}$.

\subsubsection{Solving the $c D$ Offset}

To support the picture described above, we have verified that the velocity histogram of substructure A is apparently bimodal (see Fig. 15). Indeed, as noted previously, the asymmetry index of the velocity distribution of the $R<900^{\prime \prime}$ sample is 0.996 . This suggests that there is a high-velocity population excess, and this may be partially due to substructure A. The statistics of the substructure A velocity distribution indicates a scaled tail index at 0.886 , and no significant gaps were found. We note that one of the two peaks of the velocity distribution of substructure A is centered very nearly at the velocity of the $\mathrm{cD}$ galaxy. The Dip test applied to this sample indicates that the probability of nonunimodality is $\sim 30 \%$. Both peaks also seems to have a reasonably narrow dispersion, suggesting that clump A galaxies may be separated into two subsamples: galaxies with 


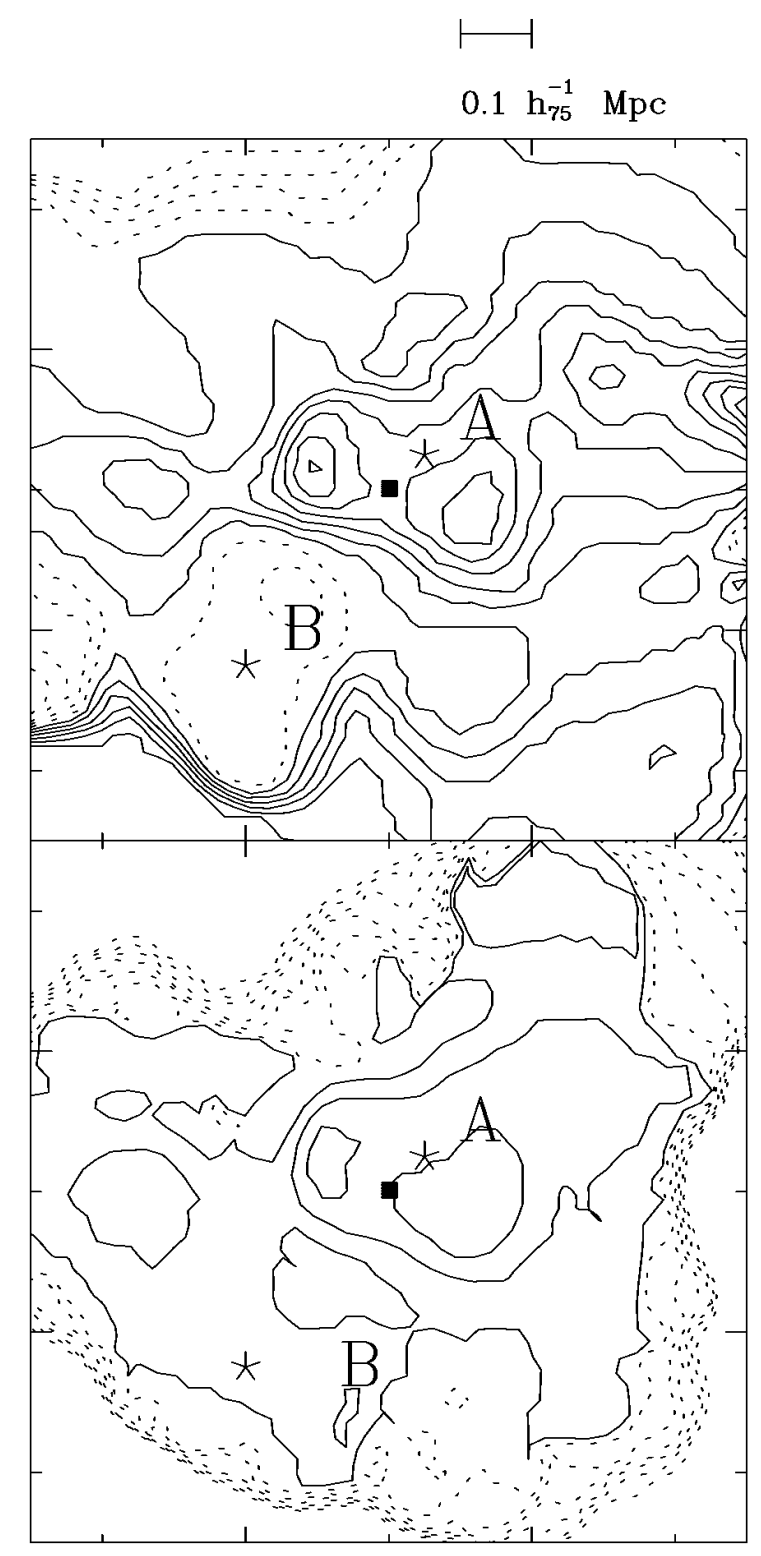

FIG. 11.-Same as Fig. 10, but showing the local average velocity contour map. Top, contours run from 13,100 to $15,700 \mathrm{~km} \mathrm{~s}^{-1}$ in intervals of $200 \mathrm{~km} \mathrm{~s}^{-1}$; bottom, contours run from 10,000 to $14,500 \mathrm{~km} \mathrm{~s}^{-1}$ in intervals of $500 \mathrm{~km} \mathrm{~s}^{-1}$.

$v<14,750 \mathrm{~km} \mathrm{~s}^{-1}$ belong to the core sample defined above, and galaxies with $v>14,750 \mathrm{~km} \mathrm{~s}^{-1}$ form the actual background group A. This group is denoted in Table 3 as $\mathrm{A}^{\prime}$.

Figure 16 shows the isodensity map that results from removing the $\mathrm{A}^{\prime}$ galaxies from the core region (this new sample is denoted in Table 3 as "Core $-\mathrm{A}^{\prime}$ "). There is no sign of any clumping at the location where substructure A was previously detected. The average velocity of this core sample is almost exactly the same as that of the $\mathrm{cD}$ galaxy $\left(14,030 \pm 42 \mathrm{~km} \mathrm{~s}^{-1}\right)$, that is, the velocity offset of the cD galaxy is contained within the $90 \%$ interval on central location in velocity space and is no longer significant (see the confidence limits in Table 3 ).

\subsubsection{The $\beta$-Problem Revisited}

The velocity dispersion for the new core sample discussed above is much smaller than the value generally quoted for A3558 (see Table 3), which, for instance, led Bardelli et al.

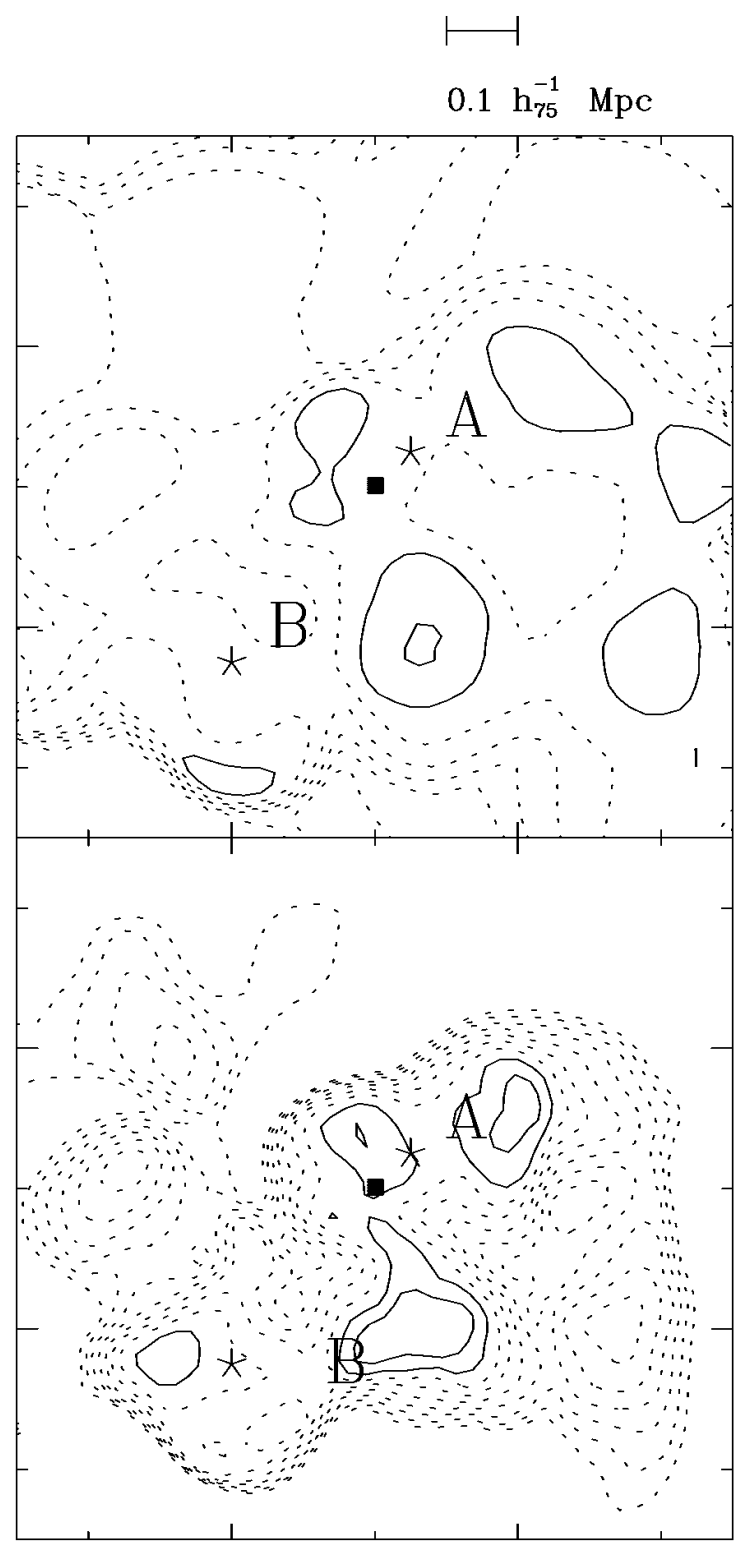

FIG. 12.-Same as Fig. 10, but showing the local velocity dispersion contour map. Top, contours run from 100 to $1300 \mathrm{~km} \mathrm{~s}^{-1}$ in intervals of $200 \mathrm{~km} \mathrm{~s}^{-1}$; bottom, contours run from 0 to $675 \mathrm{~km} \mathrm{~s}^{-1}$ in intervals of 75 $\mathrm{km} \mathrm{s}^{-1}$.

(1996) to postulate a "strong $\beta$-problem" for A3558. Assuming the new value given in Table 3 , we find $\beta_{\text {spec }}=$ $1.13_{-0.20}^{+0.18}$, to be compared with $\beta_{\text {fit }}=0.611$ estimated by Bardelli et al. (1996) from fitting the X-ray brightness to an isothermal model. A $\beta$-problem still remains, but it can no longer be considered "extreme" (the $\beta_{\text {spec }}$ value found by Bardelli et al. 1996 for A3558 is 1.79).

Nevertheless, the above results may be improved by further removing substructure B galaxies from the core sample. This removal may be justified if we interpret the $\mathrm{X}$-ray extension toward $\mathrm{B}$ as being due to the gas response to a local fluctuation of the gravitational potential due to B. This interpretation is corroborated by the WT analysis of the X-ray emission of A3558 given by Slezak, Durret, \& Gerbal (1994), which shows evidence for an X-ray counterpart for substructure B. Since the dynamical time of collision for the gas is much shorter than for the galaxies, we can 


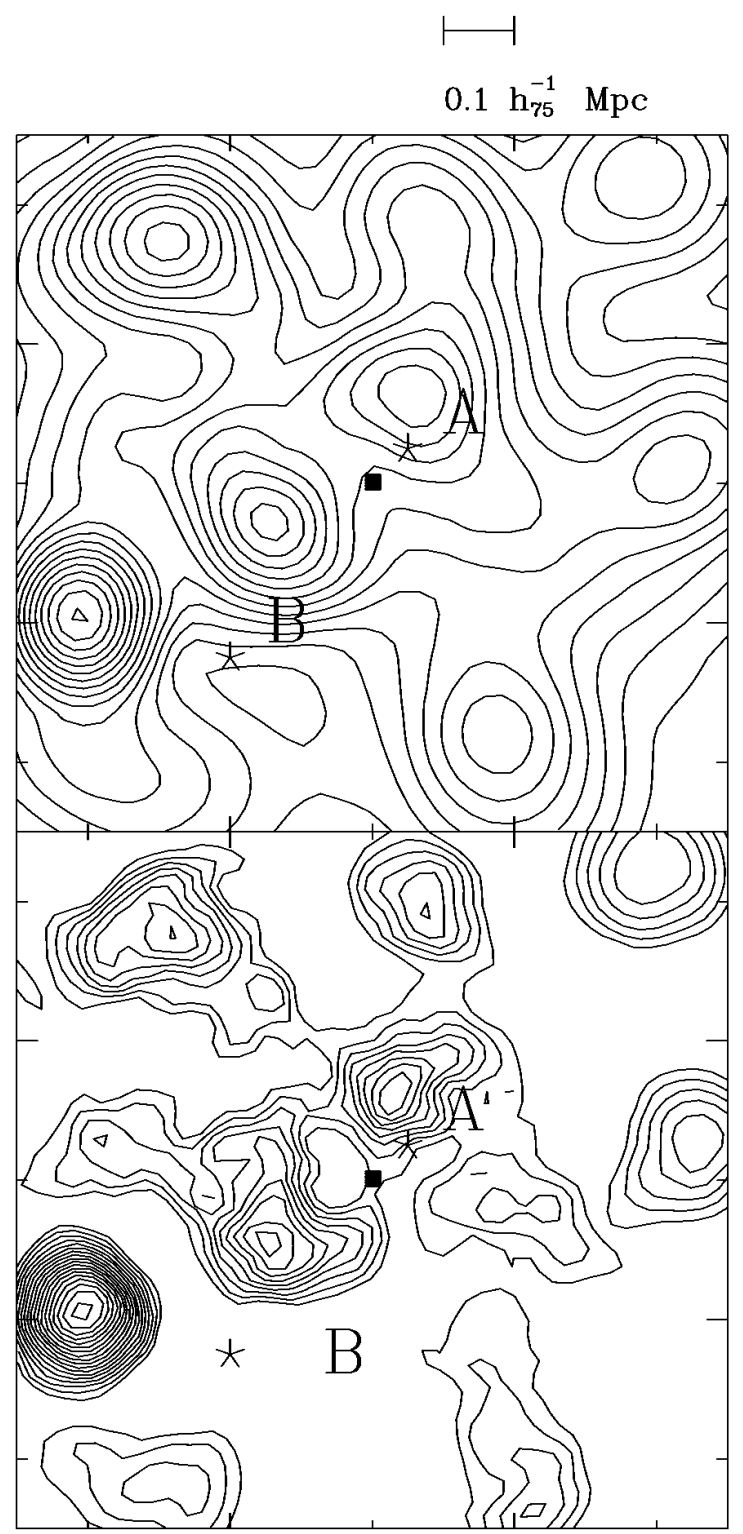

FIG. 13.- Same as Fig. 10, but for the central core sample of faint galaxies, $19<B<21$. Top, contours run from zero to $3.2 \times 10^{-4}$ galaxies $\mathrm{deg}^{-2}$ in intervals of $2 \times 10^{-5}$; bottom, contours run from zero to $1.7 \times 10^{-4}$ galaxies deg $^{-2}$ in intervals of $1 \times 10^{-5}$.

assume that the infall of subclump B galaxies is still a recent event and has not strongly disturbed the dynamics of the core. The kinematic parameters for the sample obtained by removing substructure $B$ from the core defined before are given in Table 3. Note that the difference between the $\mathrm{cD}$ velocity and the mean velocity of the core is further reduced for this sample. We now find $\beta_{\text {spec }}=0.89_{-0.23}^{+0.33}$, which is much closer to $\beta_{\mathrm{fit}}$ than the previous calculated value. Moreover, if we take into account the more reliable $A S C A$ temperatures for A3558 given by Markevitch et al. (1996), we will find that $\beta_{\text {spec }}=0.70 \pm 0.15$, a value that coincides almost exactly with the $\beta_{\text {fit }}=0.611$ estimated by Bardelli et al. Note that using the global core velocity dispersion (i.e., without removing substructure B), together with $A S C A$ temperatures, yields $\beta_{\text {spec }}=1.09 \pm 0.20$, to be compared with the value quoted by Bardelli et al. of 1.79 , using the more uncertain ROSAT temperatures (Markevitch et al. 1996).
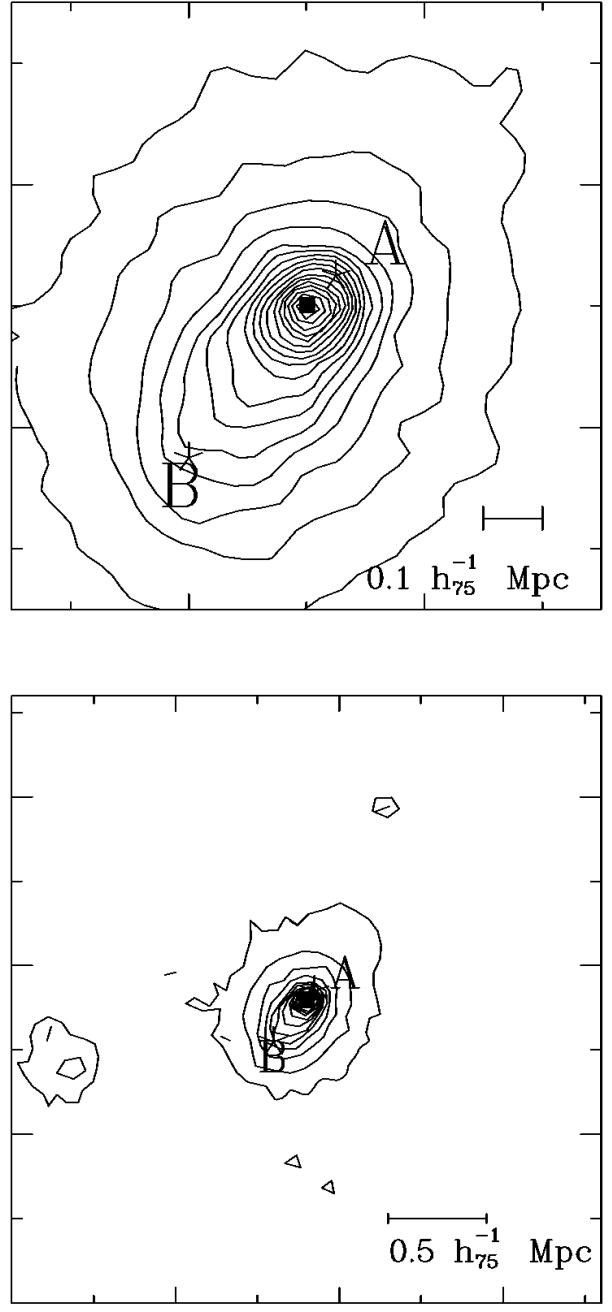

Fig. 14.-X-ray contour map of the central core region (top) and the whole field (bottom). Both maps are centered on the $\mathrm{cD}$ galaxy (square) and may be directly compared with the isodensity maps of Figs. 10 and 9, respectively.

According to Bahcall \& Lubin (1994), a solution for the $\beta$-problem arises naturally if an empirical spatial $r^{-2.4}$ law, fitted for the cluster's outer region $(>1.5 \mathrm{Mpc})$, is used instead of a King law. Since we found that the $\beta$ discrepancy for A3558 could also be naturally diminished by using a better estimation of the velocity dispersion, we analyzed in detail the density profile to check the above hypothesis. We compared the projected density profile of galaxies in the central region with the X-ray brightness profile. This profile, given by Bardelli et al. (1996), is an elliptical King law fitted to the overall cluster emission. By taking the axial ratio and position angle derived from their fit, we find that the projected central density profile of galaxies could also be very well fitted by a King law. This is in agreement with the hypothesis that our selected sample represents the relaxed core component, with the galaxies in equilibrium with the emitting gas. However, the galaxy sample selected from the whole region of our plate could only be fitted by the sum of two King profiles, one of which clearly represents the core component, whereas the other, which has a very large core radius, probably represents a background component. At the wings of the projected galaxy distribution, the resulting combined profile tends 


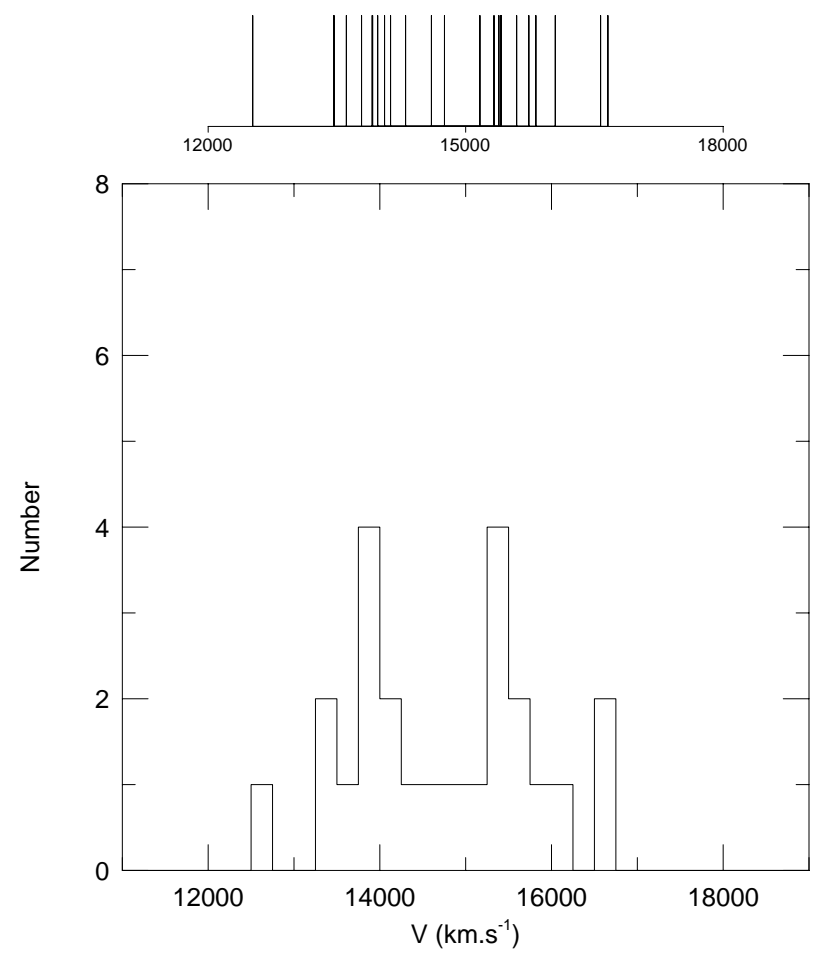

Fig. 15.-Same as Fig. 4, but for the A sample

toward an $\sim r^{-1.5}$, very similar to the one proposed by Bahcall \& Lubin.

\subsection{Mass Estimates}

We derive mass estimates for A3558 under the hypotheses that (1) the system is self-gravitating (we refer to this mass estimate as $M_{\mathrm{SG}}$; see Heisler, Tremaine \& Bahcall $1985)$ and (2) the galaxies orbit a common central potential $\left(M_{\text {sat }}\right.$; Bahcall \& Tremaine 1981). The mass estimates are calculated for increasing circular regions centered on the $\mathrm{cD}$ galaxy, after removing galaxies belonging to substructure $\mathrm{A}^{\prime}$. We have also calculated the mass of the poor cluster SC 1327-312. The resulting values are listed in Table 4 . Columns are: (1) label; (2) aperture radius in Mpc centered on the $\mathrm{CD}$ galaxy; (3) number of galaxies defining the region; (4), (5) $C_{\mathrm{BI}}$ and $S_{\mathrm{BI}}$ in $\mathrm{km} \mathrm{s}^{-1}$, respectively, together with their $90 \%$ confidence limits; (6) mass in units of $10^{14}$ $h_{75}^{-1} M_{\odot}$, according to Heisler et al. (1985), and estimated error; (7) mass in units of $10^{14} h_{75}^{-1} M_{\odot}$, according to Bahcall \& Tremaine (1981), and estimated error; and (8) $M_{\text {sat }} / L$ ratio in units of $10^{3} M_{\odot} / L_{\odot}$, the total luminosity being computed down to $B=19$.

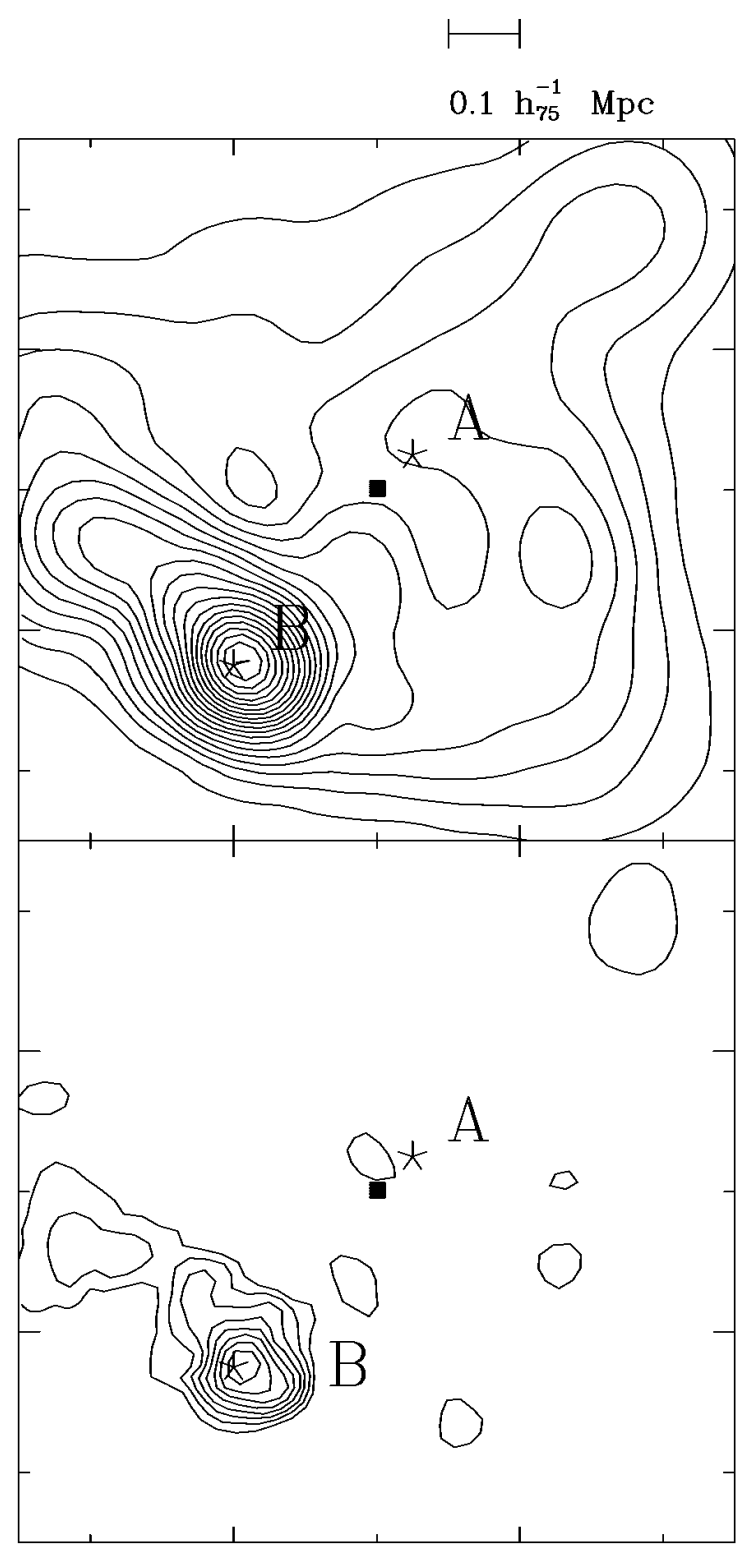

FIG. 16.-Isodensity contour map for the central core region with substructure A removed as described in the text. Contour levels are the same as in Fig. 10.

Figure 17 shows the comparison of our results with others found in the literature. Our results based on the self-gravitation hypothesis are compatible (although systematically higher) with previous dynamical estimates found by Biviano et al. (1993) and Metcalfe et al. (1987,

TABLE 4

MASS Estimates

\begin{tabular}{|c|c|c|c|c|c|c|c|}
\hline $\begin{array}{l}\text { Structure } \\
\text { (1) }\end{array}$ & $\begin{array}{c}\text { Radius } \\
\text { (2) }\end{array}$ & $\begin{array}{c}N_{\text {gal }} \\
\text { (3) }\end{array}$ & $\begin{array}{c}C_{\mathrm{BI}} \\
\left(\mathrm{km} \mathrm{s}^{-1}\right) \\
(4)\end{array}$ & $\begin{array}{c}S_{\mathrm{BI}} \\
\left(\mathrm{km} \mathrm{s}^{-1}\right) \\
(5)\end{array}$ & $\begin{array}{c}M_{\mathrm{SG}} \\
\left(10^{14} h_{75}^{-1} M_{\odot}\right) \\
(6)\end{array}$ & $\begin{array}{c}M_{\text {sat }} \\
\left(10^{14} h_{75}^{-1} M_{\odot}\right) \\
(7)\end{array}$ & $\begin{array}{c}M_{\text {sat }} / L \\
\left(10^{3} M_{\odot} / L_{\odot}\right) \\
(8)\end{array}$ \\
\hline$R<500^{\prime \prime}$ & 0.42 & 59 & $14046 \pm 106$ & $806 \pm 80$ & $3.28(1.06)$ & $1.45(0.29)$ & $0.25(0.05)$ \\
\hline$R<798^{\prime \prime}$ & 0.67 & 98 & $14065_{-90}^{+100}$ & $978_{-65}^{+64}$ & $7.58(1.91)$ & $3.05(0.55)$ & $0.27(0.05)$ \\
\hline$R<1000^{\prime \prime}$ & 0.84 & 130 & $14073 \pm 88$ & $957 \pm 54$ & $8.91(1.95)$ & $3.72(0.55)$ & $0.24(0.04)$ \\
\hline$R<1200^{\prime \prime}$ & 1.00 & 160 & $14160_{-79}^{+8}$ & $973+49$ & $10.95(2.16)$ & $4.53(0.61)$ & $0.23(0.03)$ \\
\hline SC $1327-312 \ldots \ldots$ & 0.34 & 16 & $14841_{-140}^{+139}$ & $580_{-118}^{+119}$ & $1.07(0.66)$ & $0.38(0.17)$ & $0.21(0.09)$ \\
\hline
\end{tabular}

NoTES.-Quoted uncertainties represent $90 \%$ confidence intervals. Values in parentheses are estimated errors. 


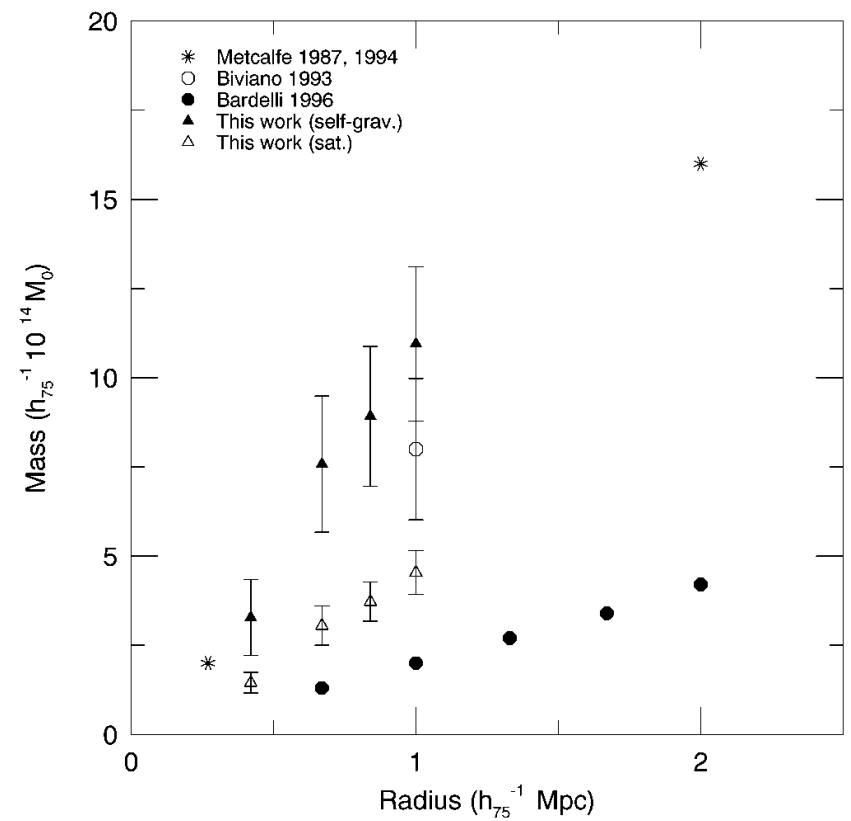

FIG. 17.- Mass estimates (in units of $10^{14} h_{75}^{-1} M_{\odot}$ ) within given aperture radii (in $\mathrm{Mpc}$ ) centered around the $\mathrm{cD}$ galaxy, according to several authors.

1994), based on different samples. Although systematically higher by a factor of $\sim 2$, our results based on the "satellite" hypothesis provide lower values for the mass estimates, which are more compatible with the mass estimates provided by Bardelli et al. (1996), based on X-ray data. This is consistent with the fact that most of the cluster mass is not bound to the visible galaxies. The discrepancy between our mass estimates compared with the X-ray total mass estimates may be partially explained by the presence of substructures that were not well taken into account in our calculations (Bird 1994). Indeed, only substructure $A^{\prime}$ could be reasonably isolated in velocity space. We note that, within the errors, the $M / L$ ratio is approximately constant with radius distance, averaging to about $250 M_{\odot} / L_{\odot}$ (the poor cluster SC $1327-312$ also has an $M / L$ ratio consistent with that of A3558). This suggests that galaxies follow the same distribution as the total cluster mass, as already noted by Bardelli et al. (1996).

\section{DISCUSSION}

In this paper, we have examined the cluster of galaxies A3558, focusing on substructure analysis, using position and velocity data. We find that a simple analysis of the velocity distribution is not sufficient to resolve the cluster into multiple components. Only a three-dimensional analysis can establish the nature of these subsystems.

The AK isodensity, isovelocity, and isodispersion maps and the WT analysis all indicate that the region defining A3558 is actually composed of a collection of several groups of galaxies, suggesting that A3558 is a dynamically complex, young cluster of galaxies. This conclusion is consistent with recent results (Bardelli et al. 1996; Markevitch et al. 1996) that utilized independent (namely, X-ray) data.

We also find a "preferential alignment" at the $\sim 45^{\circ}$ position angle of a diverse set of features in this cluster, ranging from small to large scales. In addition to this result, the number statistics of the bright X-ray clusters in the
Hydra-Centaurus region also demonstrates the presence of a linear feature that is not a chance aggregation of clusters (Raychaudhury et al. 1991). As Shapley himself noted, the supercluster that bears his name is notable as a result of "its great linear dimension, the numerous population, and distinctly elongated form" (Shapley 1930). Although standing only as a qualitative analysis, our results can be taken as observational support of an anisotropic merger scenario (West 1994a; see also Rhee, van Haarlem \& Katgert 1992; Plionis 1994). Other observational support for such a scenario comes from an analysis of correlations between the spatial distribution of substructures and the surrounding matter found in a sample of seven X-ray clusters with at least three major subclumps (West, Jones, \& Forman 1995). Other similar examples of substructure/large-scale distribution alignments are the Coma Cluster (West et al. 1995), A426 (Schwarz et al. 1992), and AWM 7 (Stern et al. 1995).

Our analysis of the cluster's core clearly indicates that the $\mathrm{cD}$ velocity offset is no longer observed. This is interesting because it is generally supposed that dominant $\mathrm{cD}$ galaxies in clusters are formed out of debris from disrupted galaxies orbiting near the center of mass of the clusters and/or by accretion of intracluster gas from a cooling flow, so their velocities should reflect that of the cluster's center of mass. Also, this result is compatible with the analysis of Gebhardt \& Beers (1991), where the authors suggested that a large fraction of clusters in their data sample that presented significant $\mathrm{cD}$ offsets could be examples of clusters with real substructure. Our results are also compatible with the study of Bird (1994) on the correlation between the cD offset and the degree of substructuring.

Concerning the $\beta$-problem, although the incompleteness in velocity data of our catalog at regions greater than 1.5 Mpc forbids a direct check of Bahcall \& Lubin's (1994) hypothesis, a background component could be responsible, at least partially, for the observed $r^{-2.4}$ law. However, only a complete velocity sample for the outer cluster region can establish whether the $r^{-2.4}$ law represents a real trend in cluster density profiles or not. Such an analysis would be extremely important for our understanding of cluster formation.

It is interesting to note that our results imply that (1) the intracluster gas and the galaxies are in approximate thermodynamic equilibrium $\left(\beta_{\mathrm{spec}}=\beta_{\mathrm{fit}}\right)$; and (2) the intracluster gas is more spread in phase space than the galaxies $\left(\beta_{\text {spec }}<1\right)$. This implies no $\beta$-discrepancy for A3558, but a mechanism to explain why $\beta_{\text {spec }}<1$ is necessary. An early satellite merger with A3558 is suggestive, since the ellipsoidal external X-ray isophotes of the cluster could be interpreted as a merger signature. Finally, our results indicate that a superestimation of the velocity dispersion due to substructure contamination is clearly an important factor contributing to the arisal of the $\beta$-discrepancy in clusters.

We thank Timothy Beers for providing the ROSTAT code and the first version of the adaptive kernel program. His valuable referee comments were also very helpful for improving the final version of this manuscript, and we thank him for that. We also thank Andrea Biviano for discussions about the bootstrapping techniques used in this work. C. C. D. acknowledges a fellowship from Fundação CAPES. This work has been partly supported by a CNPqCNRS grant. 
Abell, G. O., Corwin, H. G., Jr., \& Olowin, R. P. 1989, ApJS, 70, 1

Ashman, K. M., Bird, C., \& Zepf, S. E. 1995, AJ, 108, 2348

Bahcall, J. N., \& Tremaine, S. 1981, ApJ, 244, 805

Bahcall, N. A., \& Lubin, L. M. 1994, ApJ, 426, 513

Bardelli, S., Zucca, E., Malizia, A., Zamorani, G., Scaramella, R., \& Vettolani, G. 1996, A\&A, 305, 435

Bardelli, S., Zucca, E., Vettolani, G., Zamorani, G., Scaramella, R., Collins, C. A., \& MacGillivray, H. T. 1994, MNRAS, 267, 665

Beers, T. C., Flynn, K., \& Gebhardt, K. 1990, AJ, 100, 32

Beers, T. C., Forman, W., Huchra, J. P., Jones, C., \& Gebhardt, K. 1991, AJ, 102, 1581

Beers, T. C., Gebhardt, K., Huchra, J. P., Forman, W., Jones, C., \& Bothun, G. D. 1992, ApJ, 400, 410

Bird, C. M. 1994, Ph.D. thesis, Univ. Minnesota

Bird, C. M., \& Beers, T. 1993, AJ, 105, 1596

Biviano, A., Girardi, M., Giuricin, G., Mardirossian, F., \& Mezzetti, M.

1993, ApJ, 411, L13
Biviano, A., Durret, F., Gerbal, D., Le Fevre, O., Lobo, C., Mazure, A., Slezak, E. 1996, A\&A, 311, 95

Breen, J., Raychaudhury, S., Forman, W., \& Jones, C. 1994, ApJ, 424, 59

Dantas, C. C. 1996, Master's thesis, Inst. Nac. Pesquisas Espacias, Brazil

Dantas, C. C., de Carvalho, R. R., \& Capelato, H. V. 1994, in Proc. 29th Rencontre de Moriond, Clusters of Galaxies, ed. F. Durret, A. Mazure, \& J. Trân Thanh Vân (Gif-sur-Yvette: Editions Frontières), 385

de Carvalho, R. R., Ribeiro, A. L. B., \& Zepf, S. 1994, ApJS, 93, 47

Dressler, A., \& Shectman, S. 1988, AJ, 95, 284

Escalera, E., Biviano, A., Girardi, M., Giuricin, G., Mardirossian, F., Mazure, A., \& Mezzetti, M. 1994, ApJ, 423, 539

Fitchett, M. J. 1988, MNRAS, 230, 161

Gebhardt, K., \& Beers, T. C. 1991, ApJ, 383, 72

Geller, M. J., \& Beers, T. C. 1982, PASP, 94, 421

Gerbal, D., Durret, F., \& Lachièze-Rey, M. 1994, A\&A, 288, 746

Gorenstein, P., Fabricant, D., Topka, K., Harnden, F. R., Jr., \& Tucker, W. H. 1978, ApJ, 224, 718

Grossmann, A., \& Morlet, J. 1985, in Mathematics and Physics: Lectures on Recent Results, ed. L. Streit (Singapore: World Sci.), 1

Heisler, J., Tremaine, S., \& Bahcall, J. N. 1985, ApJ, 298, 8

Jones, C., \& Forman, W. 1984, ApJ, 276, 38

Katz, N., \& White, S. D. M. 1993, ApJ, 412, 455

Kriessler, J. R., \& Beers, T. C. 1997, AJ, 113, 80

\section{EFERENCES}

Lasker, B. M., Sturch, C. R., McLean, B. J., Russell, J. L., Jenkner, H., \& Shara, M. M. 1990, AJ, 99, 2019

Markevitch, M. L., Sarazin, C. L. \& Henriksen, M. J. 1996, ApJ, preprint

Mazure, A., et al. 1997, in preparation

Melnick, J., \& Quintana, H. 1981, A\&A, 44, 67

. 1984, AJ, 89, 1288 (MQ)

Metcalfe, N., Godwin, J. G., \& Peach, J. V. 1994, MNRAS, 267, 431

Metcalfe, N., Godwin, J. G., \& Spenser, S. D. 1987, MNRAS, 225, 581

Picard, A. 1991, Ph.D. thesis, Caltech

Plionis, M. 1994, ApJS, 95, 401

Postman, M., \& Lauer, T. R. 1995, ApJ, 440, 28 (PL)

Raychaudhury, S., Fabian, A. C., Edge, A. C., Jones, C., \& Forman, W. 1991, MNRAS, 248, 101

Rhee, G., van Haarlem, M., \& Katgert, P. 1992, AJ, 103, 1721

Richstone, D., Loeb, A., \& Turner, E. L. 1992, ApJ, 393, 477

Schwarz, R. A., Edge, A. C., Voges, W., Böhringer, H., Ebeling, H., \& Briel, U. G. 1992, A\&A, 256, L11

Shapley, H. 1930, Bull. Harvard Coll. Obs., 874, 9

Silverman, B. W. 1986, Density Estimation for Statistics and Data Analysis (London: Chapman \& Hall)

Slezak, E., Bijaoui, A., \& Mars, A. 1990, A\&A, 227, 301

Slezak, E., Durret, F., \& Gerbal, D. 1994, AJ, 108, 1996

Stein, P. 1996, A\&AS, 116, 203

Stern, C., Jones, C., Hughes, J., \& Forman, W. 1995, ApJS, submitted

Teague, P. F., Carter, D., \& Gray, P. M. 1990, ApJS, 72, 715

West, M. J. 1994a, MNRAS, 268, 79 1994b, in Proc. 29th Rencontre de Moriond, Clusters of Galaxies, ed. F. Durret, A. Mazure, \& J. Trân Thanh Vân (Gif-sur-Yvette: Editions Frontières), 23

West, M. J., \& Bothun, G. D. 1990, ApJ, 350, 36

West, M. J., Jones, C., \& Forman, W. 1995, ApJ, 451, L5

Whitmore, B. C. 1990, in Clusters of Galaxies, ed. W. R. Oegerle, M. J. Fitchett, \& L. Danly (STScI Symp. Ser., 4) (Cambridge: Cambridge Univ. Press), 139

Valdes, F. 1982, Proc. SPIE, 331, 465

Zabludoff, A. I., Geller, M. J., Huchra, J. P., \& Ramella, M. 1993, AJ, 106, 1301

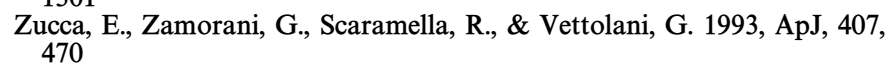

